\title{
$p$-adic cohomology: from theory to practice
}

\author{
Kiran S. Kedlaya ${ }^{1}$ \\ Introduction
}

These notes (somewhat revised from the version presented at the 2007 AWS) present a few facets of the relationship between $p$-adic analysis, algebraic de Rham cohomology, and zeta functions of algebraic varieties. A key theme is the explicit, computable nature of these constructions, which makes them suitable for numerical calculations. For instance, if you ask the computer algebra system Magma for the order of the Jacobian of a hyperelliptic curve over a field of small characteristic, this order is computed using $p$-adic cohomology. The same is true if you ask the system Sage for the $p$-adic regulator of an elliptic curve over $\mathbb{Q}$, for $p$ a good ordinary prime.

\section{Algebraic de Rham cohomology}

In this section, we introduce the notion of algebraic de Rham cohomology for smooth varieties, as originally introduced by Grothendieck [19] based on ideas of Atiyah and Hodge.

Notation 1.0.1. Throughout this section, let $K$ be a field of characteristic zero. By a "variety over $K$ ", we will mean a $K$-scheme of finite type which is reduced and separated, but not necessarily irreducible.

1.1. de Rham cohomology of smooth affine varieties. To deal with de Rham cohomology for general varieties, we will need some machinery of sheaf cohomology and hypercohomology. Before doing so, however, let us consider the case of smooth affine varieties, for which no such machinery is needed.

Definition 1.1.1. Let $R$ be a finitely generated, reduced $K$-algebra, and let $X=\operatorname{Spec} R$ be the corresponding affine variety over $K$. Let $\Omega_{R / K}$ denote the module of Kähler differentials; that is, $\Omega_{R / K}$ is the $R$-module generated by symbols $d r$ for $r \in R$, modulo the relations $d r$ for $r \in K$, and $d(a b)-a d b-b d a$ for $a, b \in R$. The module $\Omega_{R / K}$ is finitely generated over $R$, and is equipped with a derivation $d: R \rightarrow \Omega_{R / K}$ carrying $r$ to $d r$; it has the universal property that for any $K$ linear derivation $D: R \rightarrow M$ into an $R$-module, there is a unique $R$-linear map $\psi: \Omega_{R / K} \rightarrow M$ such that $D=\psi \circ d$.

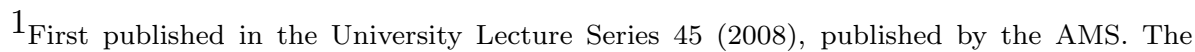
author thanks Ralf Gerkmann, Alan Lauder, Doug Ulmer, and the participants of STAGE (the Seminar on Topics in Algebra, Geometry, Etc.) for feedback on preliminary versions of these notes. The author was supported by the Southwest Center for Arithmetic Geometry (NSF grant DMS-0602287), NSF CAREER grant DMS-0545904, and a Sloan Research Fellowship.
} 
We assume hereafter that $X / K$ is smooth, which forces $\Omega_{R / K}$ to be a locally free $R$-module of rank $\operatorname{dim}(X)$. Let

$$
\Omega_{R / K}^{i}=\wedge_{R}^{i} \Omega_{R / K}
$$

be the $i$-th alternating power (or wedge power) of $\Omega_{R / K}$ over $R$. That is, $\Omega_{R / K}^{i}$ is the free $R$-module generated by symbols $\omega_{1} \wedge \cdots \wedge \omega_{i}$, modulo the submodule generated by

$$
\left(r \omega_{1}+r^{\prime} \omega_{1}^{\prime}\right) \wedge \omega_{2} \wedge \cdots \wedge \omega_{i}-r \omega_{1} \wedge \omega_{2} \wedge \cdots \wedge \omega_{i}-r^{\prime} \omega_{1}^{\prime} \wedge \omega_{2} \wedge \cdots \wedge \omega_{i}
$$

for $r, r^{\prime} \in R$, and by $\omega_{1} \wedge \cdots \wedge \omega_{i}$ whenever two of the factors are equal. Beware that the elements of the form $\omega_{1} \wedge \cdots \wedge \omega_{i}$, the so-called decomposable elements, do not comprise all of $\Omega_{R / K}^{i}$; e.g., if $R=k\left[x_{1}, x_{2}, x_{3}, x_{4}\right]$, then $d x_{1} \wedge d x_{2}+d x_{3} \wedge d x_{4}$ is a nondecomposable element of $\Omega_{R / K}^{2}$.

The map $d$ induces maps $d: \Omega_{R / K}^{i} \rightarrow \Omega_{R / K}^{i+1}$. Moreover, the composition $d \circ d$ is always zero. We thus have a complex $\Omega_{R / K}$, called the de Rham complex of $X$. The cohomology of this complex is called the (algebraic) de Rham cohomology of $X$, denoted $H_{\mathrm{dR}}^{i}(X)$; it is contravariantly functorial in $X$. Note that for $i>\operatorname{dim}(X)$, $\Omega_{R / K}^{i}=0$ and so $H_{\mathrm{dR}}^{i}(X)=0$.

EXercise 1.1.2. Put $R=K\left[x_{1}, \ldots, x_{n}\right]$, so that $X=\mathbb{A}_{K}^{n}$ is the affine $n$-space over $K$. Check that

$$
H^{0}(X)=K, \quad H^{i}(X)=0 \quad(i>0) .
$$

Terminology 1.1.3. The elements of $\Omega_{R / K}^{i}$ are referred to as $i$-forms. An $i$-form is closed if it is in the kernel of $d: \Omega_{R / K}^{i} \rightarrow \Omega_{R / K}^{i+1}$, and exact if it is the image of $d: \Omega_{R / K}^{i-1} \rightarrow \Omega_{R / K}^{i}$. In this terminology, $H_{\mathrm{dR}}^{i}(X)$ is the quotient of the space of closed $i$-forms by the subspace of exact $i$-forms.

REMARK 1.1.4. There is a construction of algebraic de Rham cohomology that allows affines which are not smooth. Roughly speaking, given a closed immersion of the given affine scheme into a smooth affine variety (e.g., an affine space), one may use the cohomology of the de Rham complex on the formal neighborhood of the image; this does not depend on the choice of the immersion. This constructed is developed by Hartshorne in $[\mathbf{2 2}]$.

\subsection{Example: an incomplete elliptic curve.}

EXAmple 1.2.1. Assume that $P(x)=x^{3}+a x+b \in K[x]$ has no repeated roots, and put

$$
R=K[x, y] /\left(y^{2}-P(x)\right),
$$

so that $X=\operatorname{Spec} R$ is the affine part of an elliptic curve over $K$ (i.e., the complete elliptic curve minus the one point at infinity). Then $H_{\mathrm{dR}}^{0}(X)=K$, and $H_{\mathrm{dR}}^{i}(X)$ vanishes for $i>1$.

The interesting space $H_{\mathrm{dR}}^{1}(X)$ is simply the cokernel of $d: R \rightarrow \Omega_{R / K}$. To describe it, we use the relation

$$
0=d\left(y^{2}-P(x)\right)=2 y d y-P^{\prime} d x
$$

in $\Omega_{R / K}$. (Throughout this example, primes denote differentiation with respect to $x$.) Since $P$ has no repeated roots, we can choose polynomials $A, B \in K[x]$ such 
that $A P+B P^{\prime}=1$. Now put

$$
\omega=A y d x+2 B d y
$$

so that

$$
d x=y \omega, \quad d y=\frac{1}{2} P^{\prime} \omega .
$$

Consequently, every element of $\Omega_{R / K}$ has a unique representation as $(C+D y) \omega$ for some $C, D \in K[x]$. For this form to be exact, there must exist $E, F \in K[x]$ such that

$$
\begin{aligned}
(C+D y) \omega & =d(E+F y) \\
& =E^{\prime} d x+F^{\prime} y d x+F d y \\
& =\left(\frac{1}{2} P^{\prime} F+F^{\prime} P\right) \omega+E^{\prime} y \omega .
\end{aligned}
$$

In particular, Dyw is always exact. As for $C \omega$, if $F$ has leading term $c x^{d}$, then $\frac{1}{2} P^{\prime} F+F^{\prime} P$ has leading term $\left(\frac{3}{2}+d\right) c x^{d+2}$. Since $\frac{3}{2}+d$ is never an integer, we can choose $c$ so that subtracting $\left(\frac{3}{2}+d\right) c x^{d+2}$ removes the leading term of $C$.

Repeating this process (of clearing leading terms from $C$ ) allows us to write $C \omega$ as an exact differential plus a $K$-linear combination of

$$
\omega, \quad x \omega .
$$

These two thus form a basis of $H_{\mathrm{dR}}^{1}(X)$.

REMARK 1.2.2. Note that by writing

$$
\omega=\frac{d x}{y}=\frac{2 d y}{P^{\prime}(x)},
$$

we can see that $\omega$ actually extends to a 1 -form on the complete elliptic curve, whereas $x \omega$ has a double pole at infinity.

REMARK 1.2.3. From the point of view of making machine computations, what is crucial here is not simply that we were able to compute the dimension of $H_{\mathrm{dR}}^{1}(X)$, or write down a basis. Rather, it is crucial that given any (closed) 1-form, we have a simple algorithm for presenting it as an exact 1-form plus a linear combination of basis elements.

Later, we will need a slight variation of the above example.

Example 1.2.4. Define $P$ as in Example 1.2.1, but this time put

$$
R=K[x, y, z] /\left(y^{2}-P(x), y z-1\right),
$$

so that $X=\operatorname{Spec} R$ is an elliptic curve over $K$ minus the point at infinity and the three points of order 2. Again, $H_{\mathrm{dR}}^{0}(X)=K$, and $H_{\mathrm{dR}}^{i}(X)$ vanishes for $i>1$.

In this case, to calculate $H_{\mathrm{dR}}^{1}(X)$, we will work not with $\omega$ but directly with $d x$. Any given element of $\Omega_{R / K}$ can be written as $(C+D y) y^{-2 i} d x$ for some nonnegative integer $i$ and some $C, D \in K[x]$. Now the relevant calculation of an exact differential (for $E, F \in K[x]$ ) is

$$
\begin{aligned}
d\left((E+F y) y^{-2 j}\right) & =E^{\prime} y^{-2 j} d x+F^{\prime} y^{-2 j+1} d x-2 j E y^{-2 j-1} d y-(2 j-1) F y^{-2 j} d y \\
& =\left(E^{\prime} P-j P^{\prime} E\right) y^{-2 j-2} d x+\left(F^{\prime} P+\frac{1-2 j}{2} P^{\prime} F\right) y^{-2 j-1} d x
\end{aligned}
$$


We interpret this as saying that in $H_{\mathrm{dR}}^{1}(X)$,

$$
\left(j P^{\prime} E\right) y^{-2 j-2} d x+\left(\frac{2 j-1}{2} P^{\prime} F\right) y^{-2 j-1} d x
$$

is cohomologous to something of the form $(G+H y) y^{-2 j} d x$.

At this point we may treat the even and odd powers of $y$ completely independently. (This is explained by the fact that the curve admits a hyperelliptic involution $x \mapsto x, y \mapsto-y$; the even and odd powers of $y$ correspond to the plus and minus eigenspaces under this involution.) In both cases, we use the fact that $P$ and $P^{\prime}$ generate the unit ideal of $K[x]$.

In the odd powers of $y$, we can reduce all the way down to $D y^{-1} d x$, then eliminate multiples of $P^{\prime} y^{-1} d x$. We are thus left with generators $d x / y, x d x / y$.

In the even powers of $y$, we can reduce all the way down to $C y^{-2} d x$, then eliminate multiples of $P y^{-2} d x$. We are thus left with generators $d x / y^{2}, x d x / y^{2}, x^{2} d x / y^{2}$.

To conclude, we have the following basis for $H_{\mathrm{dR}}^{1}(X)$ :

$$
\frac{d x}{y}, \frac{x d x}{y}, \frac{d x}{y^{2}}, \frac{x d x}{y^{2}}, \frac{x^{2} d x}{y^{2}},
$$

and again we can explicitly rewrite any 1-form in terms of these plus an exact 1-form.

EXERCISE 1.2.5. Let $P(x) \in K[x]$ be a squarefree polynomial. Compute $H_{\mathrm{dR}}^{i}(X)$ for the punctured affine line $X=\operatorname{Spec} K[x, y] /(y P(x)-1)$; again, this means that you should have an explicit recipe for presenting any 1-form as an exact 1-form plus a linear combination of basis elements.

ExERCISE 1.2.6. Repeat the derivations of Example 1.2.1 and 1.2.4 for a hyperelliptic curve $y^{2}=P(x)$. Note that the net result depends on whether $\operatorname{deg}(P)$ is odd or even; for an explanation of this, see Exercise 1.6.4.

1.3. Sheaf cohomology. In order to move past affines, we must work with sheaf cohomology and hypercohomology. We give here a rapid summary of the key points; we presume that the reader has encountered sheaf cohomology previously, e.g., in $[\mathbf{2 3}$, Chapter III]

Definition 1.3.1. Let $X$ be a scheme, and let $\mathbf{A} \mathbf{b}_{X}$ denote the category of sheaves of abelian groups on $X$. Given two complexes $C^{\cdot}=\left(0 \rightarrow C^{0} \rightarrow C^{1} \rightarrow \cdots\right)$ and $D^{*}$ in $\mathbf{A b}_{X}$, a morphism $C^{\cdot} \rightarrow D^{*}$ is a commuting diagram

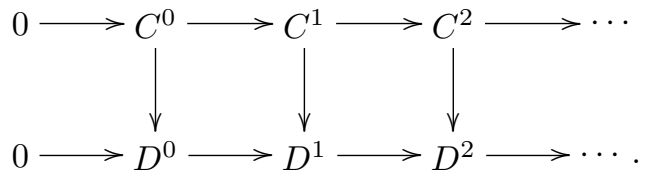

This morphism is a quasi-isomorphism if the induced maps on cohomology:

$$
\frac{\operatorname{ker}\left(C^{i} \rightarrow C^{i+1}\right)}{\operatorname{image}\left(C^{i-1} \rightarrow C^{i}\right)} \rightarrow \frac{\operatorname{ker}\left(D^{i} \rightarrow D^{i+1}\right)}{\operatorname{image}\left(D^{i-1} \rightarrow D^{i}\right)}
$$

are isomorphisms.

Definition 1.3.2. Let $\mathbf{C}$ be a full subcategory of $\mathbf{A} \mathbf{b}_{X}$ (i.e., retain some of the objects but keep all morphisms between such objects). For $C$. a complex, a resolution by $\mathbf{C}$ of $C^{\cdot}$ is a second complex $D^{\cdot}$ in $\mathbf{C}$ equipped with a quasi-isomorphism 
$C \rightarrow D^{\circ}$. As a special case, if $C \in \mathbf{A b}_{X}$ is a single object, we may identify $C$

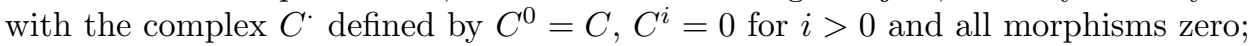
this gives the notion of a resolution by $\mathbf{C}$ of the object $C$, which is just an exact sequence $0 \rightarrow C \rightarrow D^{0} \rightarrow D^{1} \rightarrow \cdots$ with each $D^{i} \in \mathbf{C}$. (If $\mathbf{C}$ is defined by an adjective describing certain objects of $\mathbf{A} \mathbf{b}_{X}$, e.g., "injective", we will refer to an "injective resolution" instead of a "resolution by $\mathbf{C}$ ".)

Definition 1.3.3. We say $\mathscr{F} \in \mathbf{A} \mathbf{b}_{X}$ is injective if the functor $\operatorname{Hom}(\cdot, \mathscr{F})$ is exact. For any $\mathscr{F} \in \mathbf{A} \mathbf{b}_{X}$, there exists a monomorphism $\mathscr{F} \rightarrow \mathscr{G}$ with $\mathscr{G}$ injective [23, Proposition III.2.2]; this is commonly described by saying that $\mathbf{A b}_{X}$ is an abelian category which has enough injectives.

Definition 1.3.4. Since $\mathbf{A} \mathbf{b}_{X}$ has enough injectives, any $\mathscr{F}$ admits an injective resolution $\mathscr{F}$; moreover, given two injective resolutions, there is a third one to which each admits a quasi-isomorphism. We define the sheaf cohomology $H^{i}(X, \mathscr{F})$ as the $i$-th cohomology of the complex $\Gamma\left(X, \mathscr{F}^{\circ}\right)$; it turns out to be canonically independent of the choice of an injective resolution. The construction $\mathscr{F} \mapsto H^{i}(X, \mathscr{F})$ is functorial in $\mathscr{F}$, with $H^{0}(X, \cdot)$ being the global sections functor $\Gamma(X, \cdot)$. Also, given a short exact sequence $0 \rightarrow \mathscr{F} \rightarrow \mathscr{G} \rightarrow \mathscr{H} \rightarrow 0$, we obtain a long exact sequence

$$
\cdots \rightarrow H^{i}(X, \mathscr{F}) \rightarrow H^{i}(X, \mathscr{G}) \rightarrow H^{i}(X, \mathscr{H}) \stackrel{\delta_{i}}{\rightarrow} H^{i+1}(X, \mathscr{F}) \rightarrow \cdots ;
$$

the maps $\delta_{i}: H^{i}(X, \mathscr{H}) \rightarrow H^{i+1}(X, \mathscr{F})$ (which are themselves functorial in the short exact sequence) are the connecting homomorphisms. (Here $H^{-1}(X, \mathscr{H})=0$, so we start $0 \rightarrow H^{0}(X, \mathscr{F}) \rightarrow H^{0}(X, \mathscr{G}) \rightarrow \cdots$.)

Definition 1.3.5. We say $\mathscr{F}$ is acyclic if $H^{i}(X, \mathscr{F})=0$ for $i>0$. One can then show that sheaf cohomology can be computed using any acyclic resolution, not just any injective resolution. However, we must use injectives in order to define sheaf cohomology, because the notion of acyclicity is not available until after cohomology has been defined. In other words, the notion of injectivity depends only on the category $\mathbf{A} \mathbf{b}_{X}$, whereas acyclicity depends on the choice of the functor $\Gamma(X, \cdot)$ to serve as $H^{0}$.

REMARK 1.3.6. What makes the previous observation helpful is that it is quite easy to construct acyclic resolutions in many cases, using Cech complexes. The key input in the next definition is the fact that if $X$ is affine, then any quasicoherent sheaf of $\mathscr{O}_{X}$-modules on $X$ is acyclic, because the global sections functor on quasicoherent sheaves is exact.

Definition 1.3.7. Let $X$ be a separated scheme, let $\mathscr{F}$ be a quasicoherent sheaf of $\mathscr{O}_{X}$-modules on $X$, let $I$ be a finite totally ordered set, and let $\left\{U_{i}\right\}_{i \in I}$ be a cover of $X$ by open affine subschemes. Since $X$ is separated, any nonempty finite intersection of $U_{i}$ 's is again affine. (This is the algebro-geometric analogue of a "good cover" in the parlance of [6], i.e., a cover of a manifold by open subsets such that each nonempty finite intersection is contractible.) For each finite subset $J$ of $I$, put $U_{J}=\cap_{j \in J} U_{j}$ and let $j_{J}: U_{J} \hookrightarrow X$ be the implied open immersion. Then $j_{J}$ is an affine morphism, so $\left(j_{J}\right)_{*}$ is exact. Since every quasicoherent sheaf on the affine $U_{J}$ is acyclic, $\left(j_{J}\right)_{*} j_{J}^{*} \mathscr{F}$ is acyclic on $X$.

We thus obtain an acyclic resolution $\mathscr{F}$ of $\mathscr{F}$ as follows.

- The term $\mathscr{F}^{i}$ consists of the direct sum of $\left(j_{J}\right)_{*} j_{J}^{*} \mathscr{F}$ over all $(i+1)$-element subsets $J$ of $I$. 
- The map $\mathscr{F}^{i} \rightarrow \mathscr{F}^{i+1}$, applied to an element of $\mathscr{F}^{i}$ with component $x_{J}$ in $\left(j_{J}\right)_{*} j_{J}^{*} \mathscr{F}$, produces an element of $\mathscr{F}^{i+1}$ whose component in $\left(j_{J}\right)_{*} j_{J}^{*} \mathscr{F}$ with $J=\left\{j_{0}<\cdots<j_{i+1}\right\}$ is

$$
\sum_{h=0}^{i+1}(-1)^{h} x_{J \backslash\left\{j_{h}\right\}} .
$$

In particular, the sheaf cohomology of $\mathscr{F}$ is given the cohomology of the corresponding complex of global sections. In the $i$-th position, this consists of the direct sum of $\Gamma\left(U_{J}, j_{J}^{*} \mathscr{F}\right)$ over all $(i+1)$-element subsets $J$ of $I$. (Compare [23, Theorem III.4.5], [20, Proposition 1.4.1].)

1.4. Hypercohomology and de Rham cohomology. We now pass from sheaf cohomology to sheaf hypercohomology as in $[\mathbf{2 0}, \S 0.11 .4]$, then give the definition of algebraic de Rham cohomology for an arbitrary smooth variety.

Definition 1.4.1. Let $C$ be a complex in $\mathbf{A b}_{X}$. The sheaf hypercohomology of $C^{\cdot}$, denoted $\mathbb{H}^{i}\left(C^{\cdot}\right)$, is defined as the cohomology of $\Gamma\left(X, D^{\cdot}\right)$ for any acyclic resolution $D^{\cdot}$ of $C^{\cdot}$; again, this is independent of the choice of the resolution (one can always compare to an injective resolution).

To make this definition useful, one needs a good way to manufacture acyclic resolutions of complexes, rather than of individual sheaves.

REMARK 1.4.2. Suppose we can construct a double complex $D^{\cdot}$, such that the diagram

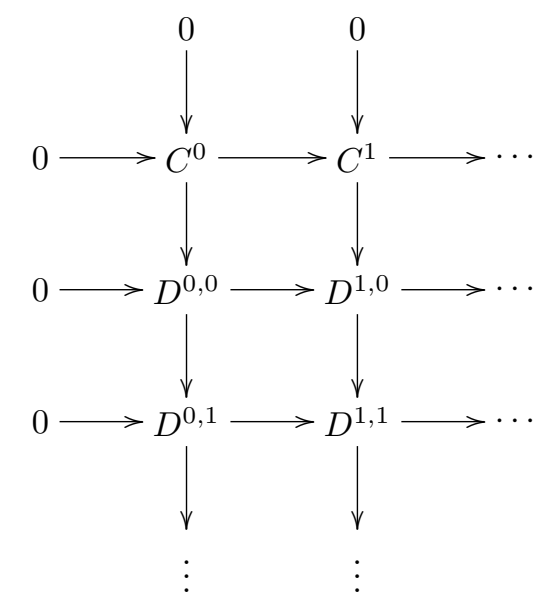

commutes, and each column gives an acyclic resolution of $C^{i}$. (In a double complex, the composition of two consecutive horizontal arrows, or two consecutive vertical arrows, must vanish.) Let $D^{\cdot}$ be the associated total complex of $D^{\circ}$,' constructed as follows.

- We take $D^{i}=\oplus_{j+k=i} D^{j, k}$.

- The map $D^{i} \rightarrow D^{i+1}$, applied to an element of $D^{i}$ with component $x_{j, k}$ in $D^{j, k}$, produces an element of $D^{i+1}$ whose component in $D^{j, k}$ is

$$
d_{\text {horizontal }}\left(x_{j-1, k}\right)+(-1)^{j} d_{\text {vertical }}\left(x_{j, k-1}\right)
$$


(The factor of $(-1)^{j}$ is needed to ensure that the composition of two of these derivations is zero; it forces the "cross terms" involving one horizontal and one vertical derivation to cancel each other out.)

Then $D$ forms an acyclic resolution of $C$.

Definition 1.4.3. Since the construction of the Cech resolution in Definition 1.3.7 is functorial in the sheaf, applying it to a complex immediately gives a diagram of the sort discussed in Remark 1.4.2.

We are now ready to give Grothendieck's definition of the algebraic de Rham cohomology of a smooth variety. (Although [19] is the source of the original definition, it is very cursory; see [23] for a fuller treatment.)

DEFINITION 1.4.4. Let $X$ be a variety over $K$. We now obtain a sheaf $\Omega_{X / K}$ of Kähler differentials, which is coherent. Let us assume further that $X$ is smooth of relative dimension $n$; then $\Omega_{X / K}$ is locally free of rank $n$. The exterior derivative is now a map of sheaves (but not of $\mathscr{O}_{X}$-modules!) $d: \mathscr{O}_{X} \rightarrow \Omega_{X / K}$, and using it we construct the de Rham complex of $X$ :

$$
0 \rightarrow \Omega_{X / K}^{0} \rightarrow \Omega_{X / K}^{1} \rightarrow \cdots \rightarrow \Omega_{X / K}^{n} \rightarrow 0 .
$$

We now define the algebraic de Rham cohomology of $X$, denoted $H_{\mathrm{dR}}^{i}(X)$, as the hypercohomology $\mathbb{H}^{i}\left(\Omega_{X / K}\right)$ of the de Rham complex; again, this is contravariantly functorial in $X$. (Formation of $H_{\mathrm{dR}}^{i}(X)$ commutes with extension of the base field, because formation of sheaf hypercohomology commutes with flat base change.)

REMARK 1.4.5. The fact that we use hypercohomology to define algebraic de Rham cohomology means that unlike in the affine case, we no longer automatically have $H_{\mathrm{dR}}^{i}(X)=0$ whenever $i>\operatorname{dim}(X)$. This will be apparent in Example 1.5.1 below.

1.5. Example: a complete elliptic curve. Let us watch the definition of algebraic de Rham cohomology in action, for a complete elliptic curve.

EXAMPLE 1.5.1. Assume again that $P(x)=x^{3}+a x+b \in K[x]$ has no repeated roots. However, now let $X$ be the complete elliptic curve $y^{2}=P(x)$, i.e.,

$$
X=\operatorname{Proj} K[X, Y, W] /\left(Y^{2} W-X^{3}-a X W^{2}-b W^{3}\right) .
$$

Since $X$ admits a one-dimensional space of everywhere holomorphic 1-forms, generated by $\omega=d x / y$, you might be tempted to think that $H_{\mathrm{dR}}^{1}(X)$ is one-dimensional. However, this is not what happens!

To compute $H_{\mathrm{dR}}^{i}(X)$, we use a simple Čech resolution. Let $U$ be the affine curve, i.e., $X$ minus the point at infinity $[0: 1: 0]$. Let $V$ be $X$ minus the three geometric points of the form $(x, 0)$, where $x$ is a root of $P$. We now find that the de Rham cohomology of $X$ is computed by the cohomology of the complex $D$, where

$$
\begin{aligned}
& D^{0}=\Gamma\left(U, \mathscr{O}_{U}\right) \oplus \Gamma\left(V, \mathscr{O}_{V}\right) \\
& D^{1}=\Gamma\left(U, \Omega_{U}\right) \oplus \Gamma\left(V, \Omega_{V}\right) \oplus \Gamma\left(U \cap V, \mathscr{O}_{U \cap V}\right) \\
& D^{2}=\Gamma\left(U \cap V, \Omega_{U \cap V}\right) .
\end{aligned}
$$

Let us start with $H_{\mathrm{dR}}^{0}(X)$; this consists of pairs $(f, g)$ with $f \in \Gamma\left(U, \mathscr{O}_{U}\right), g \in$ $\Gamma\left(V, \mathscr{O}_{V}\right)$, and $\operatorname{Res}_{U, U \cap V}(f)-\operatorname{Res}_{V, U \cap V}(g)=0$. In other words, these are just elements of $\Gamma\left(X, \mathscr{O}_{X}\right)$, and the only such elements are the constant functions. 
Next, we consider $H_{\mathrm{dR}}^{1}(X)$. First, note that the 1-cochains are triples $\left(\omega_{U}, \omega_{V}, f\right)$ with $\omega_{U} \in \Gamma\left(U, \Omega_{U}\right), \omega_{V} \in \Gamma\left(V, \Omega_{V}\right), f \in \Gamma\left(U \cap V, \mathscr{O}_{U \cap V}\right)$. The differential takes such a triple to $\operatorname{Res}_{V, U \cap V}\left(\omega_{V}\right)-\operatorname{Res}_{U, U \cap V}\left(\omega_{U}\right)-d f$.

The 1-coboundaries are expressions of the form $(d f, d g, g-f)$ with $f \in \Gamma\left(U, \mathscr{O}_{U}\right)$ and $g \in \Gamma\left(V, \mathscr{O}_{V}\right)$. Thus the projection $\left(\omega_{U}, \omega_{V}, f\right) \mapsto \omega_{U}$ induces a map

$$
H_{\mathrm{dR}}^{1}(X) \rightarrow H_{\mathrm{dR}}^{1}(U)
$$

we wish to show that this map is a bijection. We first check injectivity. If $\left(\omega_{U}, \omega_{V}, f\right)$ is a 1-cocycle on $X$, and $\omega_{U}$ is a 1-coboundary on $U$, then $\left(0, \omega_{V}, f\right)$ is also a 1cocycle on $X$, that is, $d f=\operatorname{Res}_{V, U \cap V}\left(\omega_{V}\right)$. This means that $f$ cannot have a pole at any point of $V$, as otherwise $d f$ would have at least a double pole at that point. Consequently, $\omega_{V}$ is a 1-coboundary on $V$, so $(0,0, f)$ is a 1 -cocycle on $X$. This is only possible if $f$ is constant, in which case $(0,0, f)$ is a 1-coboundary on $X$.

We now check surjectivity. By the computation we made of $H_{\mathrm{dR}}^{1}(U)$ in Example 1.2.1, it suffices to check that for any $c_{1}, c_{2} \in K$, we can find a 1-cocycle $\left(\omega_{U}, \omega_{V}, f\right)$ with $\omega_{U}=c_{1} d x / y+c_{2} x d x / y$. Namely, we take

$$
\omega_{V}=c_{1} \frac{d x}{y}+c_{2}\left(\frac{x d x}{y}-d\left(\frac{y^{2}}{x^{2}}\right)\right), \quad f=-c_{2} \frac{y^{2}}{x^{2}} .
$$

(To see that $x d x / y+d\left(y^{2} / x^{2}\right)$ is holomorphic at $\infty$, we compute in terms of the local coordinate $t=y / x$ at $\infty$, using the local expansions $x=t^{-2}+O\left(t^{0}\right)$ and $y=t^{-3}+O\left(t^{-1}\right)$.)

Finally, we compute $H_{\mathrm{dR}}^{2}(X)$. This consists of elements of $\Gamma\left(U \cap V, \Omega_{U \cap V}\right)$ modulo expressions of the form $\operatorname{Res}_{V, U \cap V}\left(\omega_{V}\right)-\operatorname{Res}_{U, U \cap V}\left(\omega_{U}\right)-d f$. By the computation we made of $H_{\mathrm{dR}}^{1}(U \cap V)$ in Example 1.2.4, we know that every element of $H_{\mathrm{dR}}^{1}(U \cap V)$ can be expressed as a $K$-linear combination of $d x / y, x d x / y, d x / y^{2}$, $x d x / y^{2}, x^{2} d x / y^{2}$. The same is true of $H_{\mathrm{dR}}^{2}(X)$, but with some redundancy: the terms $d x / y, x d x / y$ are holomorphic on $U$, so they can be absorbed by $\omega_{U}$, while the terms $d x / y^{2}, x d x / y^{2}$ are holomorphic on $V$, so they can be absorbed by $\omega_{V}$. This proves that $H_{\mathrm{dR}}^{2}(X)$ is at most one-dimensional.

To show that $H_{\mathrm{dR}}^{2}(X)$ is in fact one-dimensional, we must use some properties of residues (see Definition 1.5.2 below). Any $K$-linear combination of $d x / y, x d x / y$, $d x / y^{2}, x d x / y^{2}, x^{2} d x / y^{2}$ with a nonzero coefficient of $x^{2} d x / y^{2}$ has a simple pole at $\infty$ with nonzero residue. On the other hand, the residues at $\infty$ of the three quantities $\operatorname{Res}_{V, U \cap V}\left(\omega_{V}\right), \operatorname{Res}_{U, U \cap V}\left(\omega_{U}\right), d f$ must all vanish: the first is holomorphic at $\infty$, the third has residue zero by a local computation, and the second has residue zero because it has no other poles, and the sum of the residues at all poles must vanish.

To conclude, we find that

$$
\operatorname{dim}_{K} H_{\mathrm{dR}}^{0}(X)=1, \quad \operatorname{dim}_{K} H_{\mathrm{dR}}^{1}(X)=2, \quad \operatorname{dim}_{K} H_{\mathrm{dR}}^{2}(X)=1,
$$

in agreement with the topological Betti numbers. (This agreement is explained by a comparison theorem with topological cohomology; see Theorem 1.7.2.)

Here are the properties of residues used in the previous example. See [41] for proofs and further discussion; also see [23, Remark 7.14].

DeFinition 1.5.2. For $z$ a smooth geometric point on a curve $X$ over $K$, let $t$ be a local parameter of $X$ at $z$. Then any meromorphic 1-form $\omega$ on $X$ can be expanded around $z$ as an expression $\sum_{i=m}^{\infty} c_{i} t^{i} d t$, with $c_{i} \in \kappa(z)$. The residue of $\omega$ 
at $z$ is the value $c_{-1}$; it is independent of the choice of the parameter $t$. Moreover, if $X$ is smooth proper, then the sum of the residues of any given $\omega$ at all $z$ is zero.

Remark 1.5.3. In Example 1.5.1, we can form an injection $H^{0}\left(X, \Omega_{X / K}^{1}\right) \hookrightarrow$ $H_{\mathrm{dR}}^{1}(X)$ by converting $\omega \in \Gamma\left(X, \Omega_{X / K}^{1}\right)$ into the triple $\left(\operatorname{Res}_{X, U}(\omega), \operatorname{Res}_{X, V}(\omega), 0\right)$. This is a special case of the Hodge filtration on de Rham cohomology; see Remark 1.8.12.

\subsection{Excision in de Rham cohomology.}

REMARK 1.6.1. There is an excision exact sequence in algebraic de Rham cohomology. If $X$ is a smooth $K$-variety, $Z$ is a smooth subvariety of pure codimension $d$, and $U=X \backslash Z$, then

$$
\cdots \rightarrow H_{\mathrm{dR}}^{i-2 d}(Z) \rightarrow H_{\mathrm{dR}}^{i}(X) \rightarrow H_{\mathrm{dR}}^{i}(U) \rightarrow H_{\mathrm{dR}}^{i-2 d+1}(Z) \rightarrow \cdots
$$

Note the shift by the codimension of $Z$.

REMARK 1.6.3. There is a more general version of Remark 1.6.1 with $Z$ not required to be smooth, but one must replace the terms corresponding to $Z$ by some sort of local cohomology which will not be introduced here. However, (1.6.2) is enough to deduce the following additivity property of the Euler characteristic

$$
\chi_{\mathrm{dR}}(X)=\sum_{i}(-1)^{i} \operatorname{dim}_{K} H_{\mathrm{dR}}^{i}(X) .
$$

Namely, if $Z$ is any subvariety of $X$, then

$$
\chi_{\mathrm{dR}}(X)=\chi_{\mathrm{dR}}(Z)+\chi_{\mathrm{dR}}(X \backslash Z) .
$$

EXERCISE 1.6.4. Use (1.6.2) to show that, if $C$ is a smooth, geometrically connected, projective curve over $K, Z$ is a nonempty zero-dimensional subscheme of length $d$, and $U=C \backslash Z$, then

$$
\begin{aligned}
& \operatorname{dim}_{K} H_{\mathrm{dR}}^{0}(U)=1 \\
& \operatorname{dim}_{K} H_{\mathrm{dR}}^{1}(U)=\operatorname{dim}_{K} H_{\mathrm{dR}}^{1}(C)+d-1 \\
& \operatorname{dim}_{K} H_{\mathrm{dR}}^{2}(U)=0 .
\end{aligned}
$$

The quantity $\operatorname{dim}_{K} H_{\mathrm{dR}}^{1}(C)$ will turn out to be twice the genus of $C$, by the topological comparison (Theorem 1.7.2).

\subsection{Comparison with topological cohomology.}

Definition 1.7.1. Let $X$ be a scheme locally of finite type over $\mathbb{C}$. Then there is a functorial way to associate to $X$ a complex analytic variety $X^{\text {an }}$, called the $a n$ alytification of $X$. For instance, if $X=\operatorname{Spec} R$ with $R=\mathbb{C}\left[x_{1}, \ldots, x_{n}\right] /\left(f_{1}, \ldots, f_{n}\right)$ reduced, then $X^{\text {an }}$ is the common zero locus of $f_{1}, \ldots, f_{n}$ as an analytic subvariety of $\mathbb{C}^{n}$. See [21, Exposé XII, Théorème et definition 1.1] for a detailed construction.

The definition of algebraic de Rham cohomology is ultimately justified by the following result.

TheOREM 1.7.2 (Grothendieck). Let $X$ be a smooth variety over $\mathbb{C}$. Then there is a functorial isomorphism $H_{\mathrm{dR}}^{i}(X) \rightarrow H_{\mathrm{Betti}}^{i}\left(X^{\mathrm{an}}, \mathbb{C}\right)$. 
This is really two results bundled together. The first of these is that the Betti cohomology of $X^{\text {an }}$ is functorially isomorphic to the analytic de Rham cohomology of $X^{\text {an }}$, i.e., what you get by using the definition of algebraic de Rham cohomology but replacing the scheme with a complex analytic variety. This is a consequence of de Rham's theorem (which relates Betti cohomology to $C^{\infty}$ de Rham ccohomology) and Dolbeaut's theorem (which relates $C^{\infty}$ de Rham cohomology to analytic de Rham cohomology).

The second result is that the natural map $H_{\mathrm{dR}}^{i}(X) \rightarrow H_{\mathrm{dR}}^{i}\left(X^{\text {an }}\right)$ (i.e., view an algebraic cocycle as an analytic one) is an bijection. Since both sides of the comparison satisfy excision (Remark 1.6.1), by induction on dimension (and the fact that $X$ can always be embedded into a smooth projective variety, by Hironaka's resolution of singularities) we may reduce Theorem 1.7.2 to the case of $X$ smooth and projective. In this case, the claim becomes an instance of Serre's GAGA principle. (GAGA is an acronym for "Géométrie algébrique et géométrie analytique", the title of Serre's paper [40] introducing this principle.)

Theorem 1.7.3 (Complex-analytic GAGA). Let $X$ be a proper variety over $\mathbb{C}$.

(a) Any coherent sheaf on $X^{\text {an }}$ is the analytification of a coherent sheaf on $X$.

(b) For any coherent sheaves $\mathscr{E}, \mathscr{F}$ on $X$, any morphism $\mathscr{E}^{\text {an }} \rightarrow \mathscr{F}$ an is induced by a morphism $\mathscr{E} \rightarrow \mathscr{F}$.

(c) For any coherent sheaf $\mathscr{E}$ on $X$, with analytification $\mathscr{E}^{\text {an }}$, the natural maps $H^{i}(X, \mathscr{E}) \rightarrow H^{i}\left(X^{\text {an }}, \mathscr{E}{ }^{\text {an }}\right)$ are bijections.

Proof. For $X$ projective, proceed directly to Serre's original paper [40]. For the general case (which reduces to the projective case using Chow's lemma), see SGA1 [21, Exposé XII].

1.8. Spectral sequences and the Hodge filtration. There is a useful extra structure on de Rham cohomology given by the Hodge filtration. To explain how it arises algebraically, we must introduce spectral sequences; these will appear again later in the construction of Gauss-Manin connections.

REMARK 1.8.1. The notion of a spectral sequence is a generalization of the long exact sequence

$$
\cdots \rightarrow H^{i}\left(C_{1}^{\cdot}\right) \rightarrow H^{i}\left(C^{\cdot}\right) \rightarrow H^{i}\left(C_{2}^{\cdot}\right) \stackrel{\delta}{\rightarrow} H^{i+1}\left(C_{1}^{\cdot}\right) \rightarrow \cdots
$$

associated to a short exact sequence of complexes

$$
0 \rightarrow C_{1} \rightarrow C^{\cdot} \rightarrow C_{2}^{\cdot} \rightarrow 0 .
$$

The relevance of spectral sequences is described aptly by $[\mathbf{1 7}, \S 3.5]$ (which see for more details): "[to] someone who works with cohomology, they are essential in the same way that the various integration techniques are essential to a student of calculus."

Definition 1.8.2. A filtered complex is a decreasing sequence of complexes

$$
C^{\cdot}=F^{0} C^{\cdot} \supseteq F^{1} C^{\cdot} \supseteq \cdots \supseteq F^{n} C^{\cdot} \supseteq F^{n+1} C^{\cdot}=0 ;
$$

given a filtered complex, the associated graded complex is

$$
\operatorname{Gr} C=\oplus_{p \geq 0} \operatorname{Gr}^{p} C^{\cdot}, \quad \operatorname{Gr}^{p} C^{\cdot}=\frac{F^{p} C^{\cdot}}{F^{p+1} C^{\cdot}} .
$$


For instance, a short exact sequence as above gives a filtration with $F^{2} C=0$, $\mathrm{Gr}^{1} C^{\cdot} \cong F^{1} C^{\cdot}=C_{1}$, and $\operatorname{Gr}^{0} C^{\cdot}=C_{2}$. (Note that one could also talk about infinite filtrations, but a few statements below would have to be modified.)

EXAMPLE 1.8.3. For instance, if we start with a double complex $D^{p, q}$ and flatten it into a single complex $C^{i}=\oplus_{p+q=i} D^{p, q}$ as in Remark 1.4.2, we can filter this by taking

$$
F^{p} C^{i}=\bigoplus_{p^{\prime}+q=i, p^{\prime} \geq p} D^{p^{\prime}, q}
$$

yielding

$$
\mathrm{Gr}^{p} C^{i}=D^{p, i-p}
$$

We can also flip $p$ and $q$ to get a second filtration; these two filtrations will end up giving two distinct spectral sequences related to the cohomology of $C$.

As payback for being easy to use, spectral sequences sacrifice the computation of the entire cohomology of filtered complexes. Instead, they only compute the graded pieces of a certain filtration on the cohomology.

Definition 1.8.4. Let $Z^{q}$ and $B^{q}$ be the cocycles and coboundaries in a filtered complex $C^{q}$. The filtered cohomology is

$$
F^{p} H^{q}\left(C^{\cdot}\right)=\frac{F^{p} Z^{q}}{F^{p} B^{q}}=\operatorname{image}\left(H^{q}\left(F^{p} C^{\cdot}\right) \rightarrow H^{q}\left(C^{\cdot}\right)\right) ;
$$

note that the map $H^{q}\left(F^{p} C^{\cdot}\right) \rightarrow H^{q}\left(C^{\cdot}\right)$ need not be injective. The associated graded cohomology is

$$
\operatorname{Gr} H^{\cdot}\left(C^{\cdot}\right)=\oplus_{p, q} \operatorname{Gr}^{p} H^{q}\left(C^{\cdot}\right), \quad \operatorname{Gr}^{p} H^{q}\left(C^{\cdot}\right)=\frac{F^{p} H^{q}\left(C^{\cdot}\right)}{F^{p+1} H^{q}\left(C^{\cdot}\right)} .
$$

Definition 1.8.5. A spectral sequence is a sequence $\left\{E_{r}, d_{r}\right\}_{r=r_{0}}^{\infty}$, where each $E_{r}$ is a bigraded group

$$
E_{r}=\oplus_{p, q \geq 0} E_{r}^{p, q}
$$

and

$$
d_{r}: E_{r}^{p, q} \rightarrow E_{r}^{p+r, q-r+1}, \quad d_{r}^{2}=0
$$

is a map (usually called a differential) such that

$$
E_{r+1}^{p, q}=H^{p, q}\left(E_{r}\right)=\frac{\operatorname{ker}\left(d_{r}: E_{r}^{p, q} \rightarrow E_{r}^{p+r, q-r+1}\right)}{\operatorname{image}\left(d_{r}: E_{r}^{p-r, q+r-1} \rightarrow E_{r}^{p, q}\right)} .
$$

If at some point $E_{r}=E_{r+1}=\cdots$, we call this stable value the limit of the spectral sequence, denoted $E_{\infty}$. One also says that the sequence degenerates at $E_{r}$, and that the sequence converges to $E_{\infty}$.

REMARK 1.8.6. Pictures of spectral sequences speak louder than words. Here are the first three differentials of a spectral sequence represented diagramatically:

$$
\begin{array}{ccclcl}
E_{0}^{0,1} & E_{0}^{1,1} & E_{0}^{2,1} & E_{1}^{0,1} \stackrel{d_{1}}{\rightarrow} E_{1}^{1,1} \stackrel{d_{1}}{\rightarrow} E_{1}^{2,1} & E_{2}^{0,1} E_{2}^{1,1} E_{2}^{2,1} \\
d_{0} \uparrow & d_{0} \uparrow & d_{0} \uparrow & & & d_{2} \\
E_{0}^{0,0} & E_{0}^{1,0} & E_{0}^{2,0} & E_{1}^{0,0} \stackrel{d_{1}}{\rightarrow} E_{1}^{1,0} \stackrel{d_{1}}{\rightarrow} E_{1}^{2,0} & E_{2}^{0,0} & E_{2}^{1,0}
\end{array} E_{2}^{2,0}
$$


TheOREM 1.8.7. Let $F^{p} C$ be a filtered complex. Then there is a spectral sequence $\left\{E_{r}\right\}_{r=0}^{\infty}$ with

$$
\begin{aligned}
E_{0}^{p, q} & =\frac{F^{p} C^{p+q}}{F^{p+1} C^{p+q}} \\
E_{1}^{p, q} & =H^{p+q}\left(\operatorname{Gr}^{p} C^{\cdot}\right) \\
E_{\infty}^{p, q} & =\operatorname{Gr}^{p} H^{p+q}\left(C^{\cdot}\right) .
\end{aligned}
$$

Proof. See $[\mathbf{1 7}, \S 3.5]$ or $[\mathbf{6}, \S 14]$.

Terminology 1.8.8. In the previous theorem, one writes

$$
E_{r} \Rightarrow H^{\cdot}\left(C^{\cdot}\right)
$$

and says that the spectral sequence abuts to $H^{\cdot}\left(C^{\cdot}\right)$.

EXAMPLE 1.8.9. For instance, in the example of a short exact sequence, we have

$$
E_{1}^{0 q}=H^{q}\left(C_{2}^{\cdot}\right), \quad E_{1}^{1 q}=H^{q+1}\left(C_{1}^{\cdot}\right),
$$

$d_{1}: H^{q}\left(C_{2}\right) \rightarrow H^{q+1}\left(C_{1}\right)$ is the connecting homomorphism $\delta$, and $d_{2}=d_{3}=\cdots=0$ because the arrows always have a zero at one or both endpoints. For a filtered complex with $F^{n+1}=0$, we similarly have $E_{\infty}=E_{n+1}$.

REMARK 1.8.10. A map between filtered complexes is a quasi-isomorphism if the same is true at any single stage of the spectral sequence; the converse is also true here because our filtrations are finite, so the spectral sequence must degenerate at some stage. For instance, in the example of a short exact sequence, this is an instance of the five lemma.

EXAMPLE 1.8.11. We now apply the observation of Example 1.8.3 to the Cech complex that computes the de Rham cohomology of $X$. In the notation of Definition 1.3.7, we start with the double complex

$$
D^{p, q}=\oplus_{J} \Gamma\left(U_{J}, \Omega_{U_{J} / K}^{p}\right)
$$

where $J$ runs over all $(q+1)$-element subsets of $I$.

Suppose we filter this complex as in Example 1.8.3 with the indices as written. Using Theorem 1.8.7 gives a spectral sequence in which we first compute the sheaf cohomology of $\Omega_{X / K}^{p}$ for each $p$, i.e.,

$$
E_{1}^{p q}=H^{q}\left(X, \Omega_{X / K}^{p}\right) ;
$$

this is the Hodge-de Rham spectral sequence, and the filtration it determines on $H_{\mathrm{dR}}^{i}(X)$ is called the Hodge filtration (see Remark 1.8.12 for further discussion). It is a deep theorem that for $X$ smooth proper, the Hodge-de Rham spectral sequence degenerates already at $E_{1}$; this was originally established using analytic techniques, but can also be proved algebraically $[\mathbf{1 1}]$.

Now filter as in Example 1.8.3 but with the indices reversed. Using Theorem 1.8.7 now gives a spectral sequence with

$$
E_{1}^{p q}=\prod_{J} H_{\mathrm{dR}}^{q}\left(U_{J}\right)
$$

where $J$ runs over $(p+1)$-element subsets of $I$. Keep in mind that the $E_{\infty}$ term of the spectral sequence only computes the graded cohomology, whereas one must 
use the full Čech complex to compute the cohomology itself. Nonetheless, the information provided is often quite useful (e.g., as in Remark 1.8.10).

Remark 1.8.12. For $X$ smooth proper, the degeneration at $E_{1}$ of the Hodge-de Rham spectral sequence implies that

$$
\operatorname{Gr}^{p} H_{\mathrm{dR}}^{i}(X) \cong H^{i-p}\left(X, \Omega_{X / K}^{p}\right) .
$$

The Hodge numbers $h^{p, q}=\operatorname{dim}_{K} H^{q}\left(X, \Omega_{X / K}^{p}\right)$ satisfy some additional nice properties, such as the Serre duality symmetry satisfy the symmetry relation

$$
h^{q, p}=h^{n-q, n-p} \quad(n=\operatorname{dim}(X))
$$

and the complex conjugation symmetry

$$
h^{q, p}=h^{p, q}
$$

the latter is usually proved analytically (for compact Kähler manifolds) but can also be proved algebraically $[\mathbf{1 5}]$.

As an example of the above, we point out the short exact sequence

$$
0 \rightarrow \mathrm{Gr}^{1} H_{\mathrm{dR}}^{1}(X) \cong H^{0}\left(X, \Omega_{X / K}^{1}\right) \rightarrow H_{\mathrm{dR}}^{1}(X) \rightarrow \mathrm{Gr}^{0} H_{\mathrm{dR}}^{1}(X) \cong H^{1}\left(X, \mathscr{O}_{X}\right) \rightarrow 0
$$

where the two factors have equal dimension. In particular, if $X$ is a geometrically connected curve, then $\operatorname{dim}_{K} H_{\mathrm{dR}}^{1}(X)=2 g$ for $g=\operatorname{dim}_{K} H^{0}\left(X, \Omega_{X / K}^{1}\right)$ the genus of the curve.

1.9. Cohomology with logarithmic singularities. For various reasons (primarily the finiteness of sheaf cohomology for coherent sheaves), one prefers to manipulate cohomology on proper schemes whenever possible. In order to detect the cohomology of a nonproper scheme while doing calculations on a compactification, we may use logarithmic differentials.

Definition 1.9.1. By a smooth (proper) pair over a base $S$, we will mean a pair $(X, Z)$ in which $X$ is a smooth (proper) scheme over $S$ and $Z$ is a relative (to $S$ ) strict normal crossings divisor. Over a field, this means each component of $Z$ is smooth (no self-intersections allowed; that's the "strict" part), and the components of $Z$ always meet transversely.

Remark 1.9.2. If $(X, Z)$ is a smooth pair, then étale locally, $X$ should look like an affine space over $S$ and $Z$ should look like an intersection of coordinate hyperplanes. The converse is not quite true, but only because we chose to require $Z$ not to have self-intersections.

ExAmple 1.9.3. For instance, if $X$ is a smooth proper curve over $\mathbb{Z}_{p}$, then you can form a smooth proper pair $(X, Z)$ by taking $Z$ to be the Zariski closure in $X$ of a set of closed points of $X_{\mathbb{Q}_{p}}$ which have distinct images in $X_{\mathbb{F}_{p}}$.

Definition 1.9.4. Let $(X, Z)$ be a smooth pair over $K$. Put $U=X \backslash Z$ and let $j: U \hookrightarrow X$ be the implied open immersion. The sheaf of logarithmic differentials on $X$, denoted $\Omega_{(X, Z) / K}$, is the subsheaf of $j_{*} \Omega_{U / K}$ generated by $\Omega_{X / K}$ and by sections of the form $d f / f$, where $f$ is a regular function on some open subset $V$ of $X$ which only vanishes along components of $Z$. Again, we write $\Omega_{(X, Z) / K}^{i}$ for the $i$-th exterior power of $\Omega_{(X, Z) / K}^{1}$ over $\mathscr{O}_{X}$; this yields a logarithmic de Rham complex $\Omega_{(X, Z) / K}$. We define the logarithmic de Rham cohomology $H_{\mathrm{dR}}^{i}(X, Z)$ as the hypercohomology $\mathbb{H}^{i}\left(\Omega_{(X, Z) / K}\right)$. 
THEOREM 1.9.5. The evident map of complexes

$$
\Omega_{(X, Z) / K} \rightarrow j_{*} \Omega_{U / K}
$$

is a quasi-isomorphism. Hence we obtain an isomorphism $H_{\mathrm{dR}}^{i}(X, Z) \cong H_{\mathrm{dR}}^{i}(U)$.

Proof. The first assertion was originally proved by Deligne [10] using GAGA (Theorem 1.7.3). It is possible, and important for applications to $p$-adic cohomology, to give a completely algebraic proof, e.g., [1, Theorem 2.2.5].

The second assertion follows by considering the spectral sequence that goes from cohomology sheaves to hypercohomology (see $\S 1.8$ ).

EXERCISE 1.9.6. Prove Theorem 1.9.5 directly for the example in Exercise 1.2.5.

Remark 1.9.7. One might be tempted to deduce from Theorem 1.9.5 that for a smooth projective curve $X$ and a point $x \in X(K)$, for $U=X \backslash\{x\}$, every class in $H_{\mathrm{dR}}^{1}(X) \cong H_{\mathrm{dR}}^{1}(U)$ is represented by a 1 -form on $U$ with a logarithmic singularity at $x$. This is false; for instance, in Example 1.5.1, you need to allow either a double pole at one point (to pick up $x \omega$ ), or poles at two different points. (This is because the sum of the residues of the poles of a 1-form is always zero; see Definition 1.5.2.) A related observation is that $\Omega_{(X, Z) / K}$ is a sheaf on $X$, not on $U$, so that even if $U$ is affine one must use hypercohomology to compute $\Omega_{(X, Z) / K}$.

1.10. Example: a smooth hypersurface in projective space. We close this section by mentioning one higher-dimensional example due to Griffiths. We first need the following fact.

EXERCISE 1.10.1. Prove that the space $H_{\mathrm{dR}}^{i}\left(\mathbb{P}_{K}^{n}\right)$ is one-dimensional if $i=$ $0,2, \ldots, 2 n$ and zero otherwise. This uses the Hodge-de Rham spectral sequence plus the calculation of the cohomology $H^{q}\left(\mathbb{P}_{K}^{n}, \Omega_{\mathbb{P}_{K}^{n} / K}^{p}\right)$, as partially given by [23, Theorem III.5.1] (see also [20, §1.2.1]).

ExAmPle 1.10.2. Let $X$ be a smooth hypersurface in $\mathbb{P}_{K}^{n}$, defined by the homogeneous polynomial $P\left(x_{0}, x_{1}, \ldots, x_{n}\right)$. Then there are natural maps $H_{\mathrm{dR}}^{i}\left(\mathbb{P}_{K}^{n}\right) \rightarrow$ $H_{\mathrm{dR}}^{i}(X)$ which by the Lefschetz hyperplane theorem $[\mathbf{1 7}, \S 1.2]$ are isomorphisms for $i<n-1$ and injective for $i=n-1$. (That's actually a fact about complex manifolds, but by GAGA it transfers to the algebraic setting.) Since the cohomology of projective space is simple (Exercise 1.10.1), the only interesting cohomology group of $X$ is $H_{\mathrm{dR}}^{n-1}(X)$.

There is a short exact sequence

$$
0 \rightarrow \Omega_{\mathbb{P}^{n} / K} \rightarrow \Omega_{\left(\mathbb{P}^{n}, X\right) / K} \stackrel{\text { Res }}{\rightarrow} j_{*} \Omega_{X / K}^{+1} \rightarrow 0,
$$

where $j: X \rightarrow \mathbb{P}^{n}$ is the implied closed immersion. The map Res is a residue map, which can be described as follows: locally on $\mathbb{P}^{n}$, a section of $\Omega_{\left(\mathbb{P}^{n}, X\right) / K}$ can be written as $d f / f \wedge \omega$, where $f$ is a dehomogenized form of $P$; Res takes this section to the restriction of $\omega$ to $X$. Taking cohomology and using Theorem 1.9.5 gives a long exact sequence

$$
\cdots \rightarrow H_{\mathrm{dR}}^{i}\left(\mathbb{P}^{n}\right) \rightarrow H_{\mathrm{dR}}^{i}(U) \rightarrow H_{\mathrm{dR}}^{i-1}(X) \rightarrow H_{\mathrm{dR}}^{i+1}\left(\mathbb{P}^{n}\right) \rightarrow \cdots,
$$

where $U=\mathbb{P}^{n} \backslash X$.

The upshot of this is that if $n$ is even, then $H_{\mathrm{dR}}^{n}(U)$ is isomorphic to $H_{\mathrm{dR}}^{n-1}(X)$. If $n$ is odd, then (using Poincaré duality) $H_{\mathrm{dR}}^{n}(U)$ is isomorphic to the quotient of 
$H_{\mathrm{dR}}^{n-1}(X)$ by the (one-dimensional) image of the map $H_{\mathrm{dR}}^{n-1}\left(\mathbb{P}^{n}\right) \rightarrow H_{\mathrm{dR}}^{n-1}(X)$, the so-called primitive middle cohomology of $X$.

The point is that $U$ is affine, so you can compute its de Rham cohomology on global sections. For the recipe for doing this easily, see Griffiths $[\mathbf{1 6}, \S 4,5]$.

REMARK 1.10.3. All of the above extends easily to smooth hypersurfaces in toric varieties, providing a rich source of examples for the study of mirror symmetry. The analogue of the Griffiths recipe (attributed to Dwork-Griffiths-Katz) is described in $[\mathbf{9}, \S 5.3]$.

\section{Frobenius actions on de Rham cohomology}

In this section, we explain how to define and compute a Frobenius action on the algebraic de Rham cohomology of a smooth proper variety over a $p$-adic field with good reduction.

Notation 2.0.1. Throughout this section, let $q$ be a power of the prime $p$, let $\mathbb{Q}_{q}$ be the unramified extension of $\mathbb{Q}_{p}$ with residue field $\mathbb{F}_{q}$, and let $\mathbb{Z}_{q}$ be the integral closure of $\mathbb{Z}_{p}$ in $\mathbb{Q}_{q}$.

REMARK 2.0.2. One could in principle deal with ramified extensions of $\mathbb{Q}_{q}$ also. Our choice not to do so skirts a couple of complicating issues, including the choice of a Frobenius lift, and failure of the integral comparison theorem between de Rham and crystalline cohomology in case the absolute ramification index is greater than $p-1$.

\section{1. de Rham and crystalline cohomology.}

Definition 2.1.1. Let $(X, Z)$ be a smooth pair over $\mathbb{Z}_{q}$. Put

$$
\begin{aligned}
X_{\mathbb{Q}} & =X \times_{\operatorname{Spec} \mathbb{Z}_{q}} \operatorname{Spec} \mathbb{Q}_{q} \\
Z_{\mathbb{Q}} & =Z \times_{\operatorname{Spec} \mathbb{Z}_{q}} \operatorname{Spec} \mathbb{Q}_{q} \\
\bar{X} & =X \times_{\operatorname{Spec} \mathbb{Z}_{q}} \operatorname{Spec} \mathbb{F}_{q} \\
\bar{Z} & =Z \times_{\operatorname{Spec} \mathbb{Z}_{q}} \operatorname{Spec} \mathbb{F}_{q} \\
U & =X \backslash Z \\
U_{\mathbb{Q}} & =X \mathbb{Q} \backslash Z_{\mathbb{Q}} \\
\bar{U} & =\bar{X} \backslash \bar{Z} \\
\widehat{U} & =\text { formal completion of } U \text { along } \bar{U} .
\end{aligned}
$$

We define the relative logarithmic de Rham cohomology $H_{\mathrm{dR}}^{i}(X, Z)$ as the hypercohomology $\mathbb{H}^{i}\left(\Omega_{(X, Z) / \mathbb{Z}_{q}}\right.$ ) (where the logarithmic de Rham complex is defined as in Definition 1.9.4 replacing $K$ with $\mathbb{Z}_{q}$ ); by flat base change, the map $H_{\mathrm{dR}}^{i}(X, Z) \otimes_{\mathbb{Z}_{q}} \mathbb{Q}_{q} \rightarrow H_{\mathrm{dR}}^{i}\left(X_{\mathbb{Q}}, Z_{\mathbb{Q}}\right)$ is an isomorphism.

One then has the following comparison theorem of Berthelot [4] in the nonlogarithmic case; the logarithmic generalization is similar. (We will not define crystalline cohomology here; see [5] for the construction in the nonlogarithmic case $Z=\emptyset$, and $[\mathbf{2 7}]$ for the logarithmic case.)

THEOREM 2.1.2. There is a canonical isomorphism between $H_{\mathrm{dR}}^{i}(X, Z)$ and the logarithmic crystalline cohomology $H_{\mathrm{crys}}^{i}(\bar{X}, \bar{Z})$. 
REMARK 2.1.3. Even without the definition of crystalline cohomology, there is still an essential piece of content that one should carry away from Theorem 2.1.2. It is that the de Rham cohomology $H_{\mathrm{dR}}^{i}(X, Z)$ is functorial in the $\bmod p$ reduction $(\bar{X}, \bar{Z})$. In particular, if $\left(X^{\prime}, Z^{\prime}\right)$ is a second smooth pair and $\bar{f}: \bar{X} \rightarrow \bar{X}^{\prime}$ is a morphism carrying $\bar{Z}$ into $\bar{Z}^{\prime}$ (i.e., $\bar{f}$ induces a morphism $(\bar{X}, \bar{Z}) \rightarrow\left(\bar{X}^{\prime}, \bar{Z}^{\prime}\right)$ of smooth pairs), then $\bar{f}$ functorially induces a morphism $H_{\mathrm{dR}}^{i}\left(X^{\prime}, Z^{\prime}\right) \rightarrow H_{\mathrm{dR}}^{i}(X, Z)$. What is most surprising about this is that in order to obtain this functoriality, it is not necessary for $\bar{f}$ to lift to a morphism $f: X \rightarrow X^{\prime}$.

One crucial instance of Remark 2.1.3 is that the $q$-power Frobenius map $F_{q}$ : $\bar{X} \rightarrow \bar{X}$ induces maps $H_{\mathrm{dR}}^{i}(X, Z) \rightarrow H_{\mathrm{dR}}^{i}(X, Z)$. These satisfy the following Lefschetz trace formula.

THEOREM 2.1.4. We have

$$
\# \bar{U}\left(\mathbb{F}_{q}\right)=\sum_{i}(-1)^{i} \operatorname{Trace}\left(F_{q}, H_{\mathrm{dR}}^{i}\left(X_{\mathbb{Q}}, Z_{\mathbb{Q}}\right)\right)
$$

Proof. By Theorem 2.1.2, we may replace $H_{\mathrm{dR}}^{i}\left(X_{\mathbb{Q}}, Z_{\mathbb{Q}}\right)$ with $H_{\text {crys }}^{i}(\bar{X}, \bar{Z})$. By excision, we may reduce to the case where $Z=\emptyset$. In this case, this is a result of Katz and Messing [28].

REMARK 2.1.5. In the usual manner (e.g., as in [23, Appendix C]), Theorem 2.1.4 leads to a product formula for the zeta function

$$
\zeta(\bar{U}, T)=\exp \left(\sum_{i=1}^{\infty} \# \bar{U}\left(\mathbb{F}_{q^{i}}\right) \frac{T^{i}}{i}\right) ;
$$

namely,

$$
\zeta(\bar{U}, T)=\prod_{i} \operatorname{det}\left(1-F_{q} T, H_{\mathrm{dR}}^{i}\left(X_{\mathbb{Q}}, Z_{\mathbb{Q}}\right)\right)^{(-1)^{i+1}} .
$$

However (as in the analogous $\ell$-adic situation), there is a key difference between the case $Z=\emptyset$ and the general case. In case $Z=\emptyset$, the Riemann hypothesis component of the Weil conjectures (now a theorem of Deligne) asserts that the polynomial $\operatorname{det}\left(1-F_{q} T, H_{\mathrm{dR}}^{i}\left(X_{\mathbb{Q}}, Z_{\mathbb{Q}}\right)\right) \in \mathbb{Z}[T]$ has roots in $\mathbb{C}$ of norm $q^{-i / 2}$. In particular, since the roots for different $i$ lie in disjoint subsets of $\mathbb{C},(2.1 .6)$ uniquely determines the factors on the right side. This is no longer true for $Z$ general, though, because the roots can get mixed during the excision process.

2.2. Rigid cohomology. The definition of crystalline cohomology is not suitable for explicit calculations. Fortunately, there is a related construction that, at the expense of involving some auxiliary choices, is much more computable; it is Berthelot's rigid cohomology. The new book [35] provides a comprehensive development; here are the salient points for our purposes. (The strategy described below is essentially that of $[\mathbf{3 1}]$.)

Definition 2.2.1. Suppose that $Z$ is the inverse image of the infinity section under the morphism $f: X \rightarrow \mathbb{P}_{\mathbb{Z}_{q}}^{1}$. For $\eta \in(1, \infty]$, put

$$
U_{\eta}=\left\{x \in X_{\mathbb{Q}}^{\text {an }}:|f(x)|<\eta\right\} ;
$$

note that $U_{\infty}=U_{\mathbb{Q}}^{\text {an }}$, whereas

$$
\cap_{\eta>1} U_{\eta}=\left\{x \in X_{\mathbb{Q}}^{\mathrm{an}}:|f(x)| \leq 1\right\}
$$


is the generic fibre of the formal scheme $\widehat{U}$. Using the formalism of algebraic de Rham cohomology, we can define the de Rham cohomology $H_{\mathrm{dR}}^{i}\left(U_{\eta}\right)$ as the hypercohomology $\mathbb{H}^{i}\left(U_{\eta}, \Omega_{U_{\eta} / \mathbb{Q}_{q}}\right)$.

EXAMPLE 2.2.2. For instance, if $X=\mathbb{P}_{\mathbb{Z}_{q}}^{1}$ and $f$ is the identity map, then $U_{\infty}$ is the whole affine line over $\mathbb{Q}_{q}$, whereas $\cap_{\eta \in(1, \infty)} U_{\eta}$ is only the closed unit disc.

By Berthelot's fibration theorem, we have the following.

Theorem 2.2.3. For $1<\eta \leq \eta^{\prime} \leq \infty$, the inclusion $U_{\eta^{\prime}} \hookrightarrow U_{\eta}$ induces an isomorphism $H_{\mathrm{dR}}^{i}\left(U_{\eta^{\prime}}\right) \cong H_{\mathrm{dR}}^{i}\left(U_{\eta}\right)$. In particular, we have $H_{\mathrm{dR}}^{i}\left(U_{\eta}\right) \cong H_{\mathrm{dR}}^{i}\left(U_{\infty}\right) \cong$ $H_{\mathrm{dR}}^{i}\left(X_{\mathbb{Q}}, Z_{\mathbb{Q}}\right)$.

REMARK 2.2.4. The isomorphism $H_{\mathrm{dR}}^{i}\left(U_{\infty}\right) \cong H_{\mathrm{dR}}^{i}\left(X_{\mathbb{Q}}, Z_{\mathbb{Q}}\right)$ in Theorem 2.2.3 amounts to a rigid analytic version of the corresponding complex analytic result, which is the combination of Theorems 1.7.2 and 1.9.5. In particular, it relies on an analogue of GAGA for formal schemes [20, §5] or rigid analytic spaces [33].

REMARK 2.2.5. Without the extra hypothesis on the existence of $f$, we would have to replace the $U_{\eta}$ in Theorem 2.2.3 with a cofinal system of strict neighborhoods of the generic fibre of $\widehat{U}$. Moreover, for best results, we would have to work not with the individual $H_{\mathrm{dR}}^{i}\left(U_{\eta}\right)$ but with their direct limit as $\eta \rightarrow 1^{+}$.

More to the point, one has a comparison theorem with crystalline cohomology, which manifests as follows.

THEOREM 2.2.6. Let $\left(X^{\prime}, Z^{\prime}\right)$ be a second smooth pair over $\mathbb{Z}_{q}$ such that $Z^{\prime}$ is the inverse image of the infinity section under $f^{\prime}: X^{\prime} \rightarrow \mathbb{P}_{\mathbb{Z}_{q}}^{1}$. Suppose that $g$ : $\widehat{U} \rightarrow \widehat{U^{\prime}}$ is a morphism of formal schemes which induces a map $U_{\eta} \rightarrow U_{\eta^{\prime}}^{\prime}$ for some $\eta, \eta^{\prime} \in(1, \infty)$, and which induces a map $(\bar{X}, \bar{Z}) \rightarrow\left(\bar{X}^{\prime}, \bar{Z}^{\prime}\right)$. Using Theorem 2.2.3, obtain from this a map $H_{\mathrm{dR}}^{i}\left(X_{\mathbb{Q}}^{\prime}, Z_{\mathbb{Q}}^{\prime}\right) \rightarrow H_{\mathrm{dR}}^{i}\left(X_{\mathbb{Q}}, Z_{\mathbb{Q}}\right)$. Then this map corresponds, via Theorem 2.1.2, to the map $H_{\text {crys }}^{i}\left(\bar{X}^{\prime}, \bar{Z}^{\prime}\right) \rightarrow H_{\text {crys }}^{i}(\bar{X}, \bar{Z})$.

REMARK 2.2.7. The reader familiar with Dwork's proof of the rationality of zeta functions of varieties over finite fields [13], which also uses $p$-adic analytic methods, may wonder how those methods relate to rigid cohomology. The short answer is that there is a sort of duality between the two approaches; for more precise answers, see [2].

REMARK 2.2.8. The cohomology $H_{\mathrm{dR}}^{i}\left(U_{\eta}\right)$ in Theorem 2.2.3, or more properly its direct limit as $\eta \rightarrow 1^{+}$, may be interpreted as an instance of the "formal cohomology" of Monsky and Washnitzer $[\mathbf{3 9}, \mathbf{3 7}, \mathbf{3 8}]$. See [42] for a useful overview of that construction.

2.3. Example: an elliptic curve. It is time to make the previous construction explicit in an example, and once again we opt to consider an elliptic curve. This amounts to a paraphrase of [30]. (See also Edixhoven's course notes [14]. For $p=2$, one needs a different approach, given by Denef and Vercauteren [12].)

Example 2.3.1. Assume $p \neq 2$. Let $P(x)=x^{3}+a x+b \in \mathbb{Z}_{q}[x]$ be such that the reduction $\bar{P}(\bar{x})=\bar{x}^{3}+\overline{a x}+\bar{b} \in \mathbb{F}_{q}[x]$ has no repeated roots. Put

$$
\begin{aligned}
X & =\operatorname{Proj} \mathbb{Z}_{q}[W, X, Y] /\left(Y^{2} W-X^{3}-a X W^{2}-b W^{3}\right) \\
Z & =\operatorname{Proj} \mathbb{Z}_{q}[W, X, Y] /\left(Y^{2} W-X^{3}-a X W^{2}-b W^{3}, W Y\right),
\end{aligned}
$$


so that

$$
U_{\mathbb{Q}}=\operatorname{Spec} R, \quad R=\mathbb{Q}_{q}[x, y, z] /\left(y^{2}-x^{3}-a x-b, y z-1\right) .
$$

(Note that $(X, Z)$ form a smooth pair over $\mathbb{Z}_{q}$ as in Example 1.9.3.) We may obtain a map $f$ as in Definition 2.2 .1 by taking $f(x, y)=y+y^{-1}$.

In Example 1.2.4, we computed that $H_{\mathrm{dR}}^{1}\left(U_{\mathbb{Q}}\right)$ admits a basis over $\mathbb{Q}_{q}$ given by

$$
\frac{d x}{y}, \frac{x d x}{y}, \frac{d x}{y^{2}}, \frac{x d x}{y^{2}}, \frac{x^{2} d x}{y^{2}} .
$$

We now know that the $q$-power Frobenius on $\bar{U}$ induces a $\mathbb{Q}_{q}$-linear action on $H_{\mathrm{dR}}^{1}\left(U_{\mathbb{Q}}\right)$, and we wish to compute the matrix of this action.

To this end, we define a $\mathbb{Q}_{q}$-linear map $F_{q}: \widehat{U} \rightarrow \widehat{U}$ as follows:

$$
\begin{aligned}
& F_{q}(x)=x^{q} \\
& F_{q}(y)=y^{q}\left(1+\left(x^{3 q}+a x^{q}+b-\left(x^{3}+a x+b\right)^{q}\right) z^{2 q}\right)^{1 / 2} \\
& F_{q}(z)=z^{q}\left(1+\left(x^{3 q}+a x^{q}+b-\left(x^{3}+a x+b\right)^{q}\right) z^{2 q}\right)^{-1 / 2} .
\end{aligned}
$$

This then extends to a map $U_{\eta} \rightarrow U_{\eta^{\prime}}$ for some $\eta, \eta^{\prime}>1$, so we may apply Theorem 2.2.6 to deduce that this indeed induces the desired action on $H_{\mathrm{dR}}^{1}\left(U_{\mathbb{Q}}\right)$. Better yet, $F_{q}$ commutes with the hyperelliptic involution $y \mapsto-y$, so we may compute its action separately on the plus and minus eigenspaces of $H_{\mathrm{dR}}^{1}\left(U_{\mathbb{Q}}\right)$.

In short, we may use $F_{q}$ to compute the Frobenius action on the minus eigenspace of $H_{\mathrm{dR}}^{1}\left(U_{\mathbb{Q}}\right)$, which is equal to $H_{\mathrm{dR}}^{1}\left(X_{\mathbb{Q}}\right)$. To do this, we must rewrite the pullback $F_{q}^{*}(d x / y)=x^{q-1} F_{q}(z) d x$ as a $\mathbb{Q}_{q}$-linear combinations of $d x / y, x d x / y$ and an exact 1 -form (and likewise for $F_{q}^{*}(x d x / y)$ ). This calculation in general cannot be made exactly; for purposes of machine computation, one can only expect to work to a prescribed level of $p$-adic accuracy. So modulo some power of $p$, we write for instance

$$
F_{q}^{*}(d x / y) \equiv \sum_{i=0}^{N} \frac{Q_{i}(x) d x}{y^{2 i+1}}
$$

for some $Q_{i}(x) \in \mathbb{Q}_{q}[x]$, then uses the rules from Example 1.2.4 to rewrite this as a $\mathbb{Q}_{q}$-linear combination of $d x / y, x d x / y$ plus an exact 1 -form. If one has some information about the $p$-adic size of the reductions of the neglected terms in the expansion of $F_{q}^{*}(d x / y)$ (e.g., as in [30, Lemma 2]), one can then compute the matrix of action of $F_{q}$ on the basis $d x / y, x d x / y$ to any desired $p$-adic accuracy.

REMARK 2.3.2. For the purposes of computing the zeta function of $\bar{X}$, one ultimately wants to compute the characteristic polynomial of the matrix of action of $F_{q}$ to a certain amount of $p$-adic accuracy, which one can specify in advance. Namely, one is trying to compute some integer whose archimedean norm can be limited, so one needs only enough $p$-adic accuracy to pin down the integer uniquely in the archimedean range. (Put briefly, if one is trying to compute some $n \in \mathbb{Z}$ with $|n| \leq N$, it suffices to compute $n\left(\bmod p^{k}\right)$ for any $k>\log _{p}(2 N)$.)

REMARK 2.3.3. If $q \neq p$, one does not typically compute the action of $F_{q}$ directly. Instead, one starts with a map $F_{p}: \widehat{U} \rightarrow \widehat{U}$ which is $\mathbb{Q}_{q}$-semilinear for the 
Frobenius automorphism $\sigma$ of $\mathbb{Q}_{q}$, and acts on $x, y, z$ as follows:

$$
\begin{aligned}
& F_{p}(x)=x^{p} \\
& F_{p}(y)=y^{p}\left(1+\left(x^{3 p}+\sigma(a) x^{p}+\sigma(b)-\left(x^{3}+a x+b\right)^{p}\right) z^{2 p}\right)^{1 / 2} \\
& F_{p}(z)=z^{p}\left(1+\left(x^{3 p}+\sigma(a) x^{p}+\sigma(b)-\left(x^{3}+a x+b\right)^{p}\right) z^{2 p}\right)^{-1 / 2} .
\end{aligned}
$$

One then computes the matrix of action of $F_{p}$ on $d x / y, x d x / y$, and takes an appropriate norm of this matrix to recover the action of $F_{q}$. The fact that one can do this makes the use of $p$-adic cohomology particularly advantageous for computing zeta functions over fields of small characteristic; for example, the computer algebra system Magma uses $p$-adic cohomology to compute zeta functions of hyperelliptic curves in small characteristic. (In the elliptic case, there exist faster alternatives.)

REMARK 2.3.4. By contrast, if one wants to use $p$-adic cohomology to compute zeta functions over $\mathbb{F}_{p}$, one is forced to take $p$ not too large. The complexity estimates for the above procedure include a factor of $p$; however, it has been shown recently by David Harvey [24] that one can restructure the algorithm to improve the dependence on $p$ to a factor of $p^{1 / 2}$ times a power of $\log p$. This makes it conceivable to work with $p$ as big as $2^{64}$.

REMARK 2.3.5. The matrix of action of Frobenius has some uses beyond simply determining the zeta function; here is an interesting example due to Mazur, Stein, and Tate [36]. Following a suggestion of Katz, they give a formula for the $p$-adic (cyclotomic) canonical height of an elliptic curve over $\mathbb{Q}$ in terms of the Frobenius action on the de Rham cohomology over $\mathbb{Q}_{p}$. (This height is not the Néron local height; it is a global height with $p$-adic values, which computes the regulator term in Mazur-Tate-Teitelbaum's $p$-adic analogue of the Birch-Swinnerton-Dyer conjecture.) Using this formula, one can then compute $p$-adic canonical heights much more rapidly than any other known method; this was carried out in 2006 by a group of students (Jennifer Balakrishnan, Robert Bradshaw, David Harvey, Liang $\mathrm{Xiao})$ and is implemented in the computer algebra system Sage.

\section{Gauss-Manin connections}

In this section, we introduce the notion of a Gauss-Manin connection for a smooth proper morphism (of algebraic, complex analytic, or rigid analytic varieties). We then describe how in some cases, such a connection carries a Frobenius action which can be used to compute zeta functions.

\subsection{Connections in geometry and algebra.}

Definition 3.1.1. Let $X$ be any of the following:

- a $C^{\infty}$ real manifold;

- a complex analytic manifold;

- a smooth algebraic variety over a field of characteristic zero;

- a smooth rigid analytic variety over a field of characteristic zero.

Let $\Omega_{X}$ be the corresponding sheaf of differentials. Let $V$ be a vector bundle (coherent locally free sheaf) on $X$. A connection on $V$ is a bundle map $\nabla: V \rightarrow$ $V \otimes \Omega_{X}^{1}$ which is additive and satisfies the Leibniz rule: for any open set $U \subseteq X$, any $f \in \Gamma\left(U, \mathscr{O}_{U}\right)$ and $s \in \Gamma(U, V)$,

$$
\nabla(f s)=f \nabla(s)+s \otimes d f .
$$


A section $s$ is called horizontal if $\nabla(s)=0$.

Definition 3.1.2. Let $\nabla_{1}: V \otimes \Omega_{X}^{1} \rightarrow V \otimes \Omega_{X}^{2}$ be the map

$$
s \otimes \omega \mapsto \nabla(s) \wedge \omega+s \otimes d \omega,
$$

where $\wedge:\left(V \otimes \Omega_{X}^{1}\right) \otimes \Omega_{X}^{1} \rightarrow V \otimes \Omega_{X}^{2}$ denotes the map given by wedging the second and third factors. The curvature is the map $\nabla_{1} \circ \nabla: V \rightarrow V \otimes \Omega_{X}^{2}$; if it vanishes, we say $\nabla$ is integrable. (This is automatic if $\operatorname{dim}(X)=1$.)

REMARK 3.1.3. Here is another way to think about integrability of a connection $\nabla$. Let $z_{1}, \ldots, z_{n}$ be local coordinates for $X$ at a point $x$. Then $d z_{1}, \ldots, d z_{n}$ form a basis of $\Omega_{X}$ in some neighborhood of $x$. Form the dual basis $\frac{\partial}{\partial z_{1}}, \ldots, \frac{\partial}{\partial z_{n}}$ of tangent vector fields; we can contract $\nabla$ with the vector field $\frac{\partial}{\partial z_{i}}$ to obtain a map $V \rightarrow V$ satisfying the Leibniz rule with respect to $\frac{\partial}{\partial z_{i}}$. If you think of this as an action of $\frac{\partial}{\partial z_{i}}$ on sections of $V$, then $\nabla$ is integrable if and only if the $\frac{\partial}{\partial z_{i}}$ commute with each other.

REMARK 3.1.4. Here is the original differential-geometric interpretation of curvature. In real geometry, you can use a connection $\nabla$ to tell you how to move between fibres of the bundle in a "horizontal" fashion, i.e., parallel to the base. Even in a small neighborhood of a point, moving parallel to different paths on the base leading to the same endpoint can give different results. But if the curvature vanishes, then this discrepancy does not arise; this means that on any contractible neighborhood of $x \in X$, we can write down a basis of $V$ consisting of horizontal sections $s_{1}, \ldots, s_{n}$, and the connection is given in terms of this basis by

$$
\nabla\left(f_{1} s_{1}+\cdots+f_{n} s_{n}\right)=s_{1} \otimes d f_{1}+\cdots+s_{n} \otimes d f_{n} .
$$

It also means that given $x \in X$, parallel transport (the process of moving from one fibre to another via a horizontal path) gives a well-defined homomorphism $\rho: \pi_{1}(X, x) \rightarrow \mathrm{GL}\left(V_{x}\right)$, called the monodromy. (Differential geometers refer to integrable connections as flat connections, but for algebraic geometers this adjective is otherwise occupied.)

One can take the relationship between integrable connections and monodromy a step further.

Definition 3.1.5. Let $X$ be a connected complex manifold, and choose a point $x \in X$. A local system on $X$ is a homomorphism $\rho: \pi_{1}(X, x) \rightarrow \mathrm{GL}_{n}(\mathbb{C})$. As noted above, there is a natural functor from vector bundles equipped with integrable connections to local systems; it turns out to be an equivalence of categories.

3.2. Connections and differential equations. It is worth pointing out that for purposes of explicit calculations, it is common to work with differential equations instead of connections. This discussion is formal, so it works in any of the categories we allowed when defining connections.

Definition 3.2.1. Suppose that we are given a trivial vector bundle $V$ of rank $n$ over a subspace $S$ of the $t$-line, together with a connection on $V$, or equivalently, with an action of $\frac{d}{d t}$. A cyclic vector is a section $s$ of $V$ such that

$$
s, \frac{d}{d t} s, \ldots, \frac{d^{n-1}}{d t^{n-1}} s
$$


form a basis of $V$. Given a cyclic vector, we can describe horizontal sections as follows. We can write

$$
\frac{d^{n}}{d t^{n}} s=a_{0} s+a_{1} \frac{d}{d t} s+\cdots+a_{n-1} \frac{d^{n-1}}{d t^{n-1}} s
$$

for certain functions $a_{0}, \ldots, a_{n-1}$ on $S$. Consider an undetermined section $v$ of $V$, which must be given by

$$
v=f_{0} s+f_{1} \frac{d}{d t} s+\cdots+f_{n-1} \frac{d^{n-1}}{d t^{n-1}} s
$$

for certain functions $f_{0}, \ldots, f_{n-1}$ on $t$. For $v$ to be horizontal, we need

$$
\begin{aligned}
& 0=f_{0}^{\prime}+f_{n-1} a_{0} \\
& 0=f_{1}^{\prime}+f_{0}+f_{n-1} a_{1} \\
& \vdots \\
& 0=f_{n-1}^{\prime}+f_{n-2}+f_{n-1} a_{n-1} .
\end{aligned}
$$

Eliminating $f_{0}, \ldots, f_{n-2}$ leaves a differential equation of the form

$$
f_{n-1}^{(n)}+b_{1} f_{n-1}^{(n-1)}+\cdots+b_{n-2} f_{n-1}^{\prime}+b_{n-1} f_{n-1}=0 .
$$

Conversely, one can turn the differential equation into a first-order differential system in the usual fashion, and thus reconstruct $\nabla$.

REMARK 3.2.2. One might imagine the above construction as being a differential analogue of the passage from a matrix to its characteristic polynomial, which can be reversed (up to similarity) by forming the companion matrix of a polynomial.

\subsection{Gauss-Manin connections.}

Definition 3.3.1. Let $\pi: X \rightarrow S$ be a smooth proper morphism between objects in one of the categories we considered in Definition 3.1.1. Let $Z \subset X$ be a relative strict normal crossings divisor over $S$. Let $\Omega_{(X, Z) / S}=\Omega_{(X, Z) / K} / \pi^{*} \Omega_{S / K}$ be the sheaf of relative logarithmic differentials. Then the functor taking an open affine $U \subseteq S$ to the hypercohomology $\mathbb{H}^{i}\left(\Omega_{\left(\pi^{-1}(U), \pi^{-1}(U) \cap Z\right) / U}\right)$ turns out to be a sheaf; we call this sheaf the relative de Rham cohomology $H_{\mathrm{dR}}^{i}((X, Z) / S)$. It is a vector bundle on $S$ whose fibre at a point $b$ can be identified with $H_{\mathrm{dR}}^{i}\left(X_{b}, Z_{b}\right)$, where $X_{b}=\pi^{-1}(b)$ and $Z_{b}=X_{b} \cap Z$.

REMARK 3.3.2. The formation of the relative de Rham cohomology throws away some information: it only uses the "vertical" part of the differential operator $d$. What this means is that given a relative $i$-form $\omega \in \Omega_{(X, Z) / S}^{i}$, if one lifts $\omega$ to an absolute $i$-form $\tilde{\omega} \in \Omega_{(X, Z) / K}^{i}$ and differentiates the result, you may get something nonzero even if $\omega$ was a relative cocycle. If one projects the result into $\Omega_{(X, Z) / S}^{i} \otimes \Omega_{S / K}^{1}$, you have essentially constructed the Gauss-Manin connection. We will give a more formal construction below, but the procedure just described is how one really computes the Gauss-Manin connection in practice; see Example 3.4.1.

Definition 3.3.3. Equip the de Rham complex $\Omega_{(X, Z) / K}$ with the decreasing filtration

$$
F^{i}=\operatorname{image}\left[\Omega_{(X, Z) / K}^{-i} \otimes_{\mathscr{O}_{X}} \pi^{*}\left(\Omega_{S / K}^{i}\right) \rightarrow \Omega_{(X, Z) / K}\right]
$$


then form the corresponding spectral sequence (as in Theorem 1.8.7). The $E_{1}$ term of the result has

$$
E_{1}^{p, q}=\Omega_{S / K}^{p} \otimes_{\mathscr{O}_{S}} H_{\mathrm{dR}}^{q}((X, Z) / S) ;
$$

the algebraic Gauss-Manin connection is the differential $d_{1}: E_{1}^{0, q} \rightarrow E_{1}^{1, q}$. This construction was introduced by Katz and Oda [29], who showed that this is an integrable connection, and also that it agrees with the more traditional analytic description for a real or complex manifold.

Definition 3.3.4. Suppose we are working with real or complex manifolds, and that $S$ is contractible. Then the fibrations $X \rightarrow S$ and $Z \rightarrow S$ are trivial in the category of real manifolds, so we get a notion of horizontality for sections of $H_{\mathrm{dR}}^{i}((X, Z) / S)$. For general $S$, this gives a connection on $H_{\mathrm{dR}}^{i}((X, Z) / S)$; this is the usual Gauss-Manin connection, and Katz and Oda showed that it agrees with their algebraic construction.

REMARK 3.3.5. The differential equations corresponding to Gauss-Manin connections (via the transformation in §3.2) were introduced long before anyone had defined a connection, and so they have their own name. They are known as PicardFuchs equations; they arise by taking a homology class across different fibres and integrating against a fixed differential form on the total space. A number of classical differential equations (e.g., hypergeometric equations) arise in this fashion.

\subsection{Example: a family of elliptic curves.}

EXAmPle 3.4.1. Consider the family of smooth projective curves $\pi: X \rightarrow S$ with

$$
X=\operatorname{Proj} \mathscr{O}_{S}[X, Y, W] /\left(Y^{2} W-X^{3}-a(t) X W^{2}-b(t) W^{3}\right),
$$

and $S$ equal to the subscheme of the affine $t$-line on which $\Delta(t)=4 a^{2}+27 b^{3}$ does not vanish. Then there exist $A, B \in \Gamma\left(S, \mathscr{O}_{S}\right)[x]$ such that

$$
A P+B P_{x}=1
$$

using subscripted $x$ and $t$ for the partial derivatives in $x$ and $t$, respectively. Put

$$
\omega=A y d x+2 B d y,
$$

so that a basis for $H_{\mathrm{dR}}^{1}(X / S)$ is given by

$$
\omega, x \omega .
$$

On the other hand, if we let $Z$ be the infinity section, then $H_{\mathrm{dR}}^{1}((X, Z) / S)=$ $H_{\mathrm{dR}}^{1}(X, S)$, and it will be easier to compute the Gauss-Manin connection on the affine $U=X \backslash Z$.

In the relative module of differentials $\Omega_{U / S}^{1}$, i.e., modulo $d t$, we have as before the relation

$$
2 y d y=P_{x} d x
$$

and again

$$
d x=y \omega, \quad d y=\frac{1}{2} P_{x} \omega .
$$

But in the full module $\Omega_{U / K}^{1}$, the relation lifts to

$$
2 y d y=P_{x} d x+P_{t} d t
$$


and it is this discrepancy that gives rise to the connection. It follows that

$$
\begin{aligned}
d x \wedge d t & =y \omega \wedge d t \\
d y \wedge d t & =\frac{1}{2} P_{x} \omega \wedge d t \\
d x \wedge d y & =\frac{1}{2} P_{t} \omega \wedge d t .
\end{aligned}
$$

To compute the connection, we are supposed to lift the basis of relative cohomology to a set of forms on the total space, then differentiate, then project onto $H_{\mathrm{dR}}^{1}((X, Z) / S) \otimes \Omega_{S / K}^{1}$. First,

$$
\begin{aligned}
\nabla(\omega) & =A d y \wedge d x+A_{t} y d t \wedge d x+2 B_{x} d x \wedge d y+2 B_{t} d t \wedge d y \\
& =\left(B_{x} P_{t}-\frac{1}{2} A P_{t}-A_{t} P-B_{t} P_{x}\right) \omega \wedge d t
\end{aligned}
$$

Second,

$$
\begin{aligned}
\nabla(x \omega) & =A x d y \wedge d x+A_{t} x y d t \wedge d x+2\left(x B_{x}+B\right) d x \wedge d y+2 x B_{t} d t \wedge d y \\
& =\left(x B_{x} P_{t}+B P_{t}-\frac{1}{2} x A P_{t}-x A_{t} P-x B_{t} P_{x}\right) \omega \wedge d t
\end{aligned}
$$

We then rewrite the quantities being wedged with $d t$ as exact relative differentials plus a linear combination of $\omega, x \omega$ as in Example 2.3.1.

If you prefer, here is another way of describing essentially the same calculation.

ExAmPLE 3.4.2. Consider the same situation as in Example 3.4.1, but now let us redefine

$$
\omega=\frac{d x}{y} .
$$

(Remember that this agrees with $A y d x+2 B d y$ only modulo $d t$.) Again, use $\omega, x \omega$ as the basis of relative differentials. These have poles along $y=0$, but never mind that; we can still compute

$$
\begin{aligned}
\nabla(\omega) & =d\left(\frac{d x}{y}\right) \\
& =-\frac{d y \wedge d x}{y^{2}} \\
& =\frac{P_{t}}{2 y^{2}} \frac{d x}{y} \wedge d t .
\end{aligned}
$$

To eliminate the pole, find the unique $C, D, E, F, G \in \Gamma\left(S, \mathscr{O}_{S}\right)$ (so these are functions of $t$ alone) such that

$$
P_{t}=a_{t} x+b_{t}=(C x+D) P+\left(E x^{2}+F x+G\right) P_{x} .
$$

Then in relative de Rham cohomology,

$$
\begin{aligned}
\frac{P_{t} d x}{2 y^{3}} & =\frac{(C x+D) d x}{2 y}+\frac{\left(E x^{2}+F x+G\right) P_{x} d x}{2 y^{3}} \\
& \equiv \frac{(C x+D) d x}{2 y}+\frac{(2 E x+F) d x}{y}
\end{aligned}
$$


Similarly,

$$
\begin{aligned}
\nabla(x \omega) & =d\left(\frac{x d x}{y}\right) \\
& =\frac{x P_{t}}{2 y^{2}} \frac{d x}{y} \wedge d t
\end{aligned}
$$

and writing

$$
x P_{t}=(H x+I) P+\left(J x^{2}+K x+L\right) P_{x},
$$

we get

$$
\frac{x P_{t} d x}{2 y^{3}} \equiv \frac{(H x+I) d x}{2 y}+\frac{(2 J x+K) d x}{y} .
$$

3.5. Gauss-Manin connections and Frobenius. The reason why GaussManin connections are relevant in rigid cohomology is that they can be used to compute Frobenius actions en masse.

EXercise 3.5.1. Let Spec $A$ be a smooth affine $\mathbb{Z}_{q}$-scheme. Let $\hat{A}$ be the $p$-adic completion of $A$, and put $\bar{A}=A / p A$. Let $\phi: \hat{A} \rightarrow \hat{A}$ be a $q$-power Frobenius lift on $\hat{A}$; that is, $\phi$ acts on $\bar{A}$ by the $q$-power map. Prove that for each $\bar{x} \in(\operatorname{Spec} \bar{A})\left(\mathbb{F}_{q}\right)$, there is a unique $x \in(\operatorname{Spec} \hat{A})\left(\mathbb{Z}_{q}\right)$ which specializes to $\bar{x}$, such that $\phi(x)=x$.

Definition 3.5.2. With notation as in Exercise 3.5.1, we call $x$ the Teichmüller lift of $\bar{x}$ with respect to $\phi$. If $x$ is a Teichmüller lift of a point not specified, we say $x$ is a Teichmüller point.

EXERCISE 3.5.3. State and prove a generalization of Exercise 3.5.1 to the case where the residue field is perfect but not necessarily finite, and/or the $p$-adic field is not necessarily unramified.

TheOrem 3.5.4 (Berthelot). Let $S=\operatorname{Spec} A$ be a smooth affine $\mathbb{Z}_{q}$-scheme. Let $\phi$ be a q-power Frobenius lift on $\hat{A}$. Let $\pi: X \rightarrow S$ be a smooth proper morphism, and put $\mathscr{E}=H_{\mathrm{dR}}^{i}\left(X_{\mathbb{Q}} / S_{\mathbb{Q}}\right)$ as a vector bundle equipped with the Gauss-Manin connection. Then there exists an isomorphism $F: \phi^{*} \mathscr{E} \cong \mathscr{E}$ of vector bundles with integrable connection on the affinoid space $\hat{S}^{\text {an }}$ (the generic fibre of $\hat{S}=\operatorname{Spf} \hat{A}$ ), such that for any positive integer $a$, and any Teichmüller point $x \in \hat{S}^{\mathrm{an}}\left(\mathbb{Q}_{q^{a}}\right)$, $F^{a}:\left(\phi^{a}\right)^{*} \mathscr{E} \cong \mathscr{E}$ induces the $q^{a}$-power Frobenius action on $H_{\mathrm{dR}}^{i}\left(X_{x}\right)$.

REMark 3.5.5. The key feature of Theorem 3.5.4 is that the Frobenius action commutes with the action of the connection; this constraint can be interpreted as a differential equation on the Frobenius action. Let's see what this looks like for $S$ a subscheme of the affine $t$-line and $\mathscr{E}$ admitting a basis $s_{1}, \ldots, s_{n}$. Define the matrices $A, N$ by

$$
\begin{aligned}
F s_{j} & =\sum_{i} A_{i j} s_{i} \\
\frac{d}{d t} s_{j} & =\sum_{i} N_{i j} s_{i} .
\end{aligned}
$$

Then the compatibility between Frobenius and the connection is equivalent to the equation

$$
N A+\frac{d}{d t} A=\left(\frac{d \phi(t)}{d t}\right) A \phi(N)
$$


given $N$, this constitutes a system of linear differential equations on the entries of A.

REMARK 3.5.7. Theorem 3.5.4 forms the basis of another method for using $p$-adic cohomology to compute zeta functions, originally proposed by Lauder [34]. Let us explain how this works in the example of a family of elliptic curves, as in Example 3.4.1. (A version of this has been worked out in detail by Hubrechts [25], and is implemented in Magma.)

In order to start this method, we must already have the Frobenius matrix on $H_{\mathrm{dR}}^{i}\left(X_{x}\right)$ for a single Teichmüller point $x$, to use as an initial condition. Say for simplicity that this point is at $t=0$. In (3.5.6), we now have the value of $A(0)$. We then compute the unique matrix $U \in M_{n \times n}\left(\mathbb{Q}_{q} \llbracket t \rrbracket\right)$ with $U(0)=I_{n}$ such that $N U+\frac{d}{d t}(U)=0$; we then obtain a solution of (3.5.6) given by

$$
N=-\frac{d}{d t}(U) U^{-1}, \quad A=U A(0) \phi(U)^{-1} .
$$

Although $U$ only converges on the open unit disc (by a result of Dwork), the matrix $A$ has better convergence properties: it has bounded coefficients, and modulo any power of $p$ its entries are the power series representing certain rational functions with no poles in $S$. If one can control the number of zeroes and poles of these rational functions, one can reconstruct a $p$-adic approximation to $A$, and then evaluate at any other Teichmüller point.

REMARK 3.5.8. One way to gain control of the degrees of the rational functions appearing in Remark 3.5.7 is to understand how the Frobenius structure behaves in discs over which the fibration has one or more singular fibres. We will discuss a particularly simple instance of this in the next section.

\section{Beyond good reduction}

So far, we have only allowed consideration of varieties over $\mathbb{Q}_{q}$ with good reduction. In this section, we explore what happens when we relax ths restriction slightly.

4.1. Semistable reduction and logarithmic connections. To keep things simple, we will work only over one-dimensional base spaces.

Definition 4.1.1. Let $(X, Z)$ be a smooth pair with $X$ one-dimensional. A logarithmic connection on $X$ is a bundle map $\nabla: V \rightarrow V \otimes \Omega_{(X, Z)}$ that satisfies the Leibniz rule.

Definition 4.1.2. Let $\nabla$ be a logarithmic connection on $(X, Z)$. For each $z \in Z, \nabla$ induces a linear map $V_{z} \rightarrow V_{z}$, called the residue of $\nabla$ at $z$, as follows. For a section $s$ specializing to a given point $\bar{s} \in V_{z}$, write $d s=f \frac{d t}{t}$ for $f$ a section of $V$ and $t$ a local parameter for $z$. Then the residue map carries $\bar{s}$ to $\bar{f}$, the specialization of $f$ to $V_{z}$.

Definition 4.1.3. Let $\pi: X \rightarrow S$ be a proper, flat, generically smooth morphism (in any of the categories from Definition 3.1.1) with $S$ one-dimensional. We say $\pi$ is semistable at $z \in S$ if the fibre $X_{z}$ is a reduced divisor with simple (but not necessarily strict) normal crossings. That is, étale locally, $S$ looks like Spec $k[t]$ and $X$ looks like Spec $k\left[x_{1}, \ldots, x_{n}\right] /\left(x_{1} \cdots x_{m}-t\right)$ for some $m, n$ (where $m$ varies from point to point). 
ExAmPle 4.1.4. For example, the Legendre family of elliptic curves

$$
y^{2}=x(x-1)(x-\lambda)
$$

is smooth over $\lambda \notin\{0,1, \infty\}$, and semistable at $\lambda=0$ and $\lambda=1$. To check at $\infty$, we reparametrize $\mu=\lambda^{-1}, x=X / \mu, y=Y / \mu^{2}$ to get

$$
Y^{2}=\mu X(X-\mu)(X-1),
$$

which means that the fibre at $\lambda=\infty$ is not reduced.

If we instead consider the family

$$
y^{2}=x(x-1)\left(x-\lambda^{2}\right),
$$

this family is indeed semistable everywhere.

By a suitable variation of the Katz-Oda arguments [29], one obtains the following.

TheOREM 4.1.5. Let $\pi: X \rightarrow S$ be a semistable morphism, smooth over $U \subseteq S$. Then the Gauss-Manin connection on $H_{\mathrm{dR}}^{i}\left(\pi^{-1}(U) / U\right)$ extends to a logarithmic connection on $S$ with nilpotent residue maps.

EXERCISE 4.1.6. Check that the Gauss-Manin connection for the Legendre family extends to a logarithmic connection on all of $\mathbb{P}^{1}$, but the residue map at infinity cannot be made nilpotent. (Hint: you can cheat by looking up this calculation in $[42, \S 7]$.

4.2. Frobenius actions on singular connections. Berthelot gave the following refinement of Theorem 3.5.4.

TheOREM 4.2.1 (Berthelot). Let $S=\operatorname{Spec} A$ be a smooth affine $\mathbb{Z}_{q}$-scheme. Let $\phi$ be a q-power Frobenius lift on $\hat{A}$. Let $\pi: X \rightarrow S$ be a proper morphism smooth over an open dense subscheme $U \subseteq S$. Put $\mathscr{E}=H_{\mathrm{dR}}^{i}\left(\pi^{-1}\left(U_{\mathbb{Q}}\right) / U_{\mathbb{Q}}\right)$ as a vector bundle equipped with the Gauss-Manin connection. Then the isomorphism $F: \phi^{*} \mathscr{E} \cong \mathscr{E}$ over $\hat{U}^{\text {an }}$ given by Theorem 3.5 .4 extends to a space of the form

$$
\left\{x \in U_{\mathbb{Q}}^{\text {an }}:|f(x)| \geq \eta\right\}
$$

for some $f \in A$ and some $\eta \in(0,1)$.

REMARK 4.2.2. To clarify, let us suppose $S=\mathbb{A}_{\mathbb{Z}_{q}}^{1}$ and let us consider the open unit disc in $S^{\text {an }}$. Then Theorem 4.2.1 gives us an isomorphism $\phi^{*} \mathscr{E} \cong \mathscr{E}$ on some annulus $A_{\eta}=\left\{t \in \mathbb{A}_{\mathbb{Q}_{q}}^{1}: \eta<|t|<1\right\}$. For applications to machine computations, one would like to be able to predict the value of $\eta$. This is complicated in general, but there is a particular case in which it is comparatively easy; see below.

Lemma 4.2.3. Let $\mathscr{E}$ be a vector bundle on the open unit t-disc over $\mathbb{Q}_{q}$, equipped with a logarithmic connection with nilpotent residue at $t=0$ and no other poles. Suppose there exists an isomorphism $\phi^{*} \mathscr{E} \rightarrow \mathscr{E}$ on some $A_{\eta}$, where $\phi: \Gamma\left(A_{\eta^{\prime}}, \mathscr{O}\right) \rightarrow$ $\Gamma\left(A_{\eta}, \mathscr{O}\right)$ for some $\eta^{\prime}<\eta \in(0,1)$ satisfies $\left.\phi(t)-t^{q} \in p \widehat{\mathbb{Z}_{q}((t)}\right)$. (Note that $\phi$ does not have to be defined on the entire open unit disc.) Then $\mathscr{E}$ is a successive extension of copies of the trivial bundle with connection (i.e., the bundle is $\mathscr{O}$ and the connection is just the exterior derivative).

Proof. The existence of the Frobenius structure ensures that the differential module $\mathscr{E}$ is solvable at 1 , so that we may deduce the claim from [32, Lemma 3.6.2]. 
REMARK 4.2.4. Resuming now Remark 4.2.2, now suppose that $\pi$ is semistable and that its only singular fibre over the open unit disc is at $t=0$. Define the matrices $N, A$ as in Remark 3.5.5. Then there is a unique matrix $U \in M_{n \times n}\left(\mathbb{Q}_{q} \llbracket t \rrbracket[\log t]\right)$ with $U \equiv I_{n}(\bmod (t, \log t))$ such that $N U+\frac{d}{d t}(U)=0$. From Lemma 4.2.3, one may deduce that each power series appearing in $U$ and $U^{-1}$ converges in the entire open unit disc. Define

$$
\phi(\log t)=q \log t+\sum_{i=1}^{\infty} \frac{(-1)^{i-1}}{i}\left(\phi(t) / t^{q}-1\right)^{i}
$$

and put $B=U^{-1} A \phi(U)$. Then (3.5.6) implies $\frac{d}{d t}(B)=0$, so $B \in M_{n \times n}\left(\mathbb{Q}_{q}\right)$.

By writing $A=U B \phi\left(U^{-1}\right)$, we deduce the following. Suppose that $\eta \in[0,1)$ has the property that $\left|\phi(t) / t^{q}-1\right|_{\eta}<1$; note that this implies $|\phi(t)|_{\eta}=\eta^{q}<1$. (Here $|\cdot|_{\eta}$ is the $\eta$-Gauss norm, i.e., $\left|\sum_{i} c_{i} t^{i}\right|_{\eta}=\sup _{i}\left\{\left|c_{i}\right| \eta^{i}\right\}$.) Then $A$ converges on $\eta \leq|t|<1$.

For explicit computations, one needs effective convergence bounds in the above argument. These are provided by results of Christol and Dwork [7].

REMARK 4.2.5. Continuing as in Remark 4.2.4, we obtain on $H_{\mathrm{dR}}^{i}\left(X_{0}\right)$ a Frobenius action $\Phi$ as well as a nilpotent operator $N$. It is natural to ask for a geometric interpretation of these; one expects them to coincide with the corresponding operators (Frobenius and monodromy) on the Hyodo-Kato cohomology $H_{\mathrm{HK}}^{i}\left(\bar{X}_{0}\right)$ via the comparison isomorphism

$$
H_{\mathrm{dR}}^{i}\left(X_{0}\right) \cong H_{\mathrm{HK}}^{i}\left(\bar{X}_{0}\right)
$$

(These notions are introduced in [26]; a similar construction in topology is the "Milnor fibre".) We do not have a proof of this coincidence, though it is strongly suggested by results of Coleman and Iovita [8] and Grosse-Klönne [18].

Note that if we wish to transfer the Frobenius action from $X_{0}$ to another fibre $X_{t}$ with $|t|<1$, we must at some point evaluate $\log (t)$, which requires choosing a branch of the $p$-adic logarithm. This is consistent with the fact that for $t \neq 0$, the HyodoKato isomorphism $H_{\mathrm{dR}}^{i}\left(X_{t}\right) \cong H_{\mathrm{HK}}^{i}\left(\bar{X}_{0}\right)$ depends on such a branch choice; the monodromy operator transfers canonically to $H_{\mathrm{dR}}^{i}\left(X_{t}\right)$, but the Frobenius operator on $H_{\mathrm{dR}}^{i}\left(X_{t}\right)$ does depend on the branch choice. 



\section{Bibliography}

[1] T.G. Abbott, K.S. Kedlaya, and D. Roe, Bounding Picard numbers of surfaces using $p$ adic cohomology, arXiv:math.NT/0601508 (version of 18 Jan 2007), to appear in Arithmetic, Geometry and Coding Theory (AGCT 2005), Societé Mathématique de France.

[2] F. Baldassarri and P. Berthelot, On Dwork cohomology for singular hypersurfaces, Geometric Aspects of Dwork Theory, Vol. I, de Gruyter, Berlin, 2004, 177-244.

[3] P. Berthelot, Cohomologie Cristalline des Schémas de Caractéristique $p>0$, Lecture Notes in Math. 407, Springer-Verlag, Berlin, 1974.

[4] P. Berthelot, Géométrie rigide et cohomologie des variétés algébriques de caractéristique $p$, Introductions aux cohomologies p-adiques (Luminy, 1984), Mém. Soc. Math. France 23 (1986), 7-32.

[5] P. Berthelot and A. Ogus, Notes on Crystalline Cohomology, Princeton Univ. Press, Princeton, 1978.

[6] R. Bott and L.W. Tu, Differential Forms in Algebraic Topology, corrected third printing, Graduate Texts in Math. 82, Springer-Verlag, 1995.

[7] G. Christol and B. Dwork, Effective p-adic bounds at regular singular points, Duke Math. J. 62 (1991), 689-720.

[8] R. Coleman and A. Iovita, The Frobenius and monodromy operators for curves and abelian varieties, Duke Math. J. 97 (1999), 171-215.

[9] D.A. Cox and S. Katz, Mirror Symmetry and Algebraic Geometry, Math. Surveys and Monographs 68, Amer. Math. Soc., 1999.

[10] P. Deligne, Équations Différentielles à Points Singuliers Réguliers, Lecture Notes in Math. 163, Springer-Verlag, Berlin, 1970.

[11] P. Deligne and L. Illusie, Relèvements modulo $p^{2}$ et dècomposition du complexe de de Rham, Invent. Math. 89 (1987), 247-270.

[12] J. Denef and F. Vercauteren, An extension of Kedlaya's algorithm to hyperelliptic curves in characteristic 2, J. Cryptology 19 (2006), 1-25.

[13] B. Dwork, On the rationality of the zeta function of an algebraic variety, Amer. J. Math. 82 (1960), 631-648.

[14] B. Edixhoven, Point counting after Kedlaya, course notes at http://www.math.leidenuniv.nl/ ${ }^{\text {edix/oww/mathof crypt/. }}$

[15] T. Ekedahl, On the multiplicative properties of the de Rham-Witt complex I, II, Ark. Mat. 22(1984), 185-239; ibid. 23 (1985), 53-102.

[16] P. Griffiths, On the periods of certain rational integrals. I, II, Annals of Math. 90 (1969), 460-495, 496-541.

[17] P. Griffiths and J. Harris, Principles of Algebraic Geometry, John Wiley \& Sons, 1978.

[18] E. Grosse-Klönne, Frobenius and monodromy operators in rigid analysis, and Drinfel'd's symmetric space, J. Alg. Geom. 14 (2005), 391-437.

[19] A. Grothendieck, On the de Rham cohomology of algebraic varieties, Publ. Math. IHÉS 29 (1966), 95-103.

[20] A. Grothendieck, Élements de géométrie algébrique III: Étude cohomologique des faisceaux cohérents, première partie (EGA 3-1), Publ. Math. IHÉS 11 (1961), 5-167.

[21] A. Grothendieck et al, Revêtements Étales et Groupe Fondamental (SGA 1), revised version, Société Mathématique de France, 2003.

[22] R. Hartshorne, On the De Rham cohomology of algebraic varieties, Publ. Math. IHÉS 45 (1975), 5-99.

[23] R. Hartshorne, Algebraic Geometry, Graduate Texts in Math. 52, Springer, 1977. 
[24] D. Harvey, Kedlaya's algorithm in larger characteristic, Int. Math. Res. Notices (2007), article ID rnm095.

[25] H. Hubrechts, Point counting in families of hyperelliptic curves, to appear in Found. Comp. Math.

[26] O. Hyodo and K. Kato, Semi-stable reduction and crystalline cohomology with logarithmic poles, Périodes p-adiques (Bures-sur-Yvette, 1988), Astérisque 223 (1994), 221-268.

[27] K. Kato, Logarithmic structures of Fontaine-Illusie, Algebraic Analysis, Geometry, and Number Theory (Baltimore, MD, 1988), Johns Hopkins Univ. Press, Baltimore, 1989, 191-224.

[28] N.M. Katz and W. Messing, Some consequences of the Riemann hypothesis for varieties over finite fields, Invent. Math. 23 (1974), 73-77.

[29] N.M. Katz and T. Oda, On the differentiation of de Rham cohomology classes with respect to parameters. J. Math. Kyoto Univ. 8 (1968), 199-213.

[30] K.S. Kedlaya, Counting points on hyperelliptic curves using Monsky-Washnitzer cohomology, J. Ramanujan Math. Soc. 16 (2001), 323-336; errata, ibid. 18 (2003), 417-418.

[31] K.S. Kedlaya, Computing zeta functions via p-adic cohomology, Algorithmic Number Theory, Lecture Notes in Comp. Sci. 3076, Springer, 2004, 1-17.

[32] K.S. Kedlaya, Semistable reduction for overconvergent F-isocrystals, I: Unipotence and logarithmic extensions, Compos. Math. 143 (2007), 1164-1212.

[33] U. Köpf, Über eigentliche Familien algebraischer Varietäten über affinoiden Räumen, Schr. Math. Inst. Univ. Münster Heft 7 (1974).

[34] A.G.B. Lauder, Deformation theory and the computation of zeta functions, Proc. London Math. Soc. 88 (2004), 565-602.

[35] B. le Stum, Rigid Cohomology, Cambridge Univ. Press, 2007.

[36] B. Mazur, W. Stein, and J. Tate, Computation of $p$-adic heights and log convergence, Doc. Math. Extra Vol. (2006), 577-614.

[37] P. Monsky, Formal cohomology. II: The cohomology sequence of a pair, Annals of Math. 88 (1968), 218-238.

[38] P. Monsky, Formal cohomology. III: Fixed point theorems, Annals of Math. 93 (1971), 315343.

[39] P. Monsky and G. Washnitzer, Formal cohomology. I, Annals of Math. 88 (1968), 181-217.

[40] J.-P. Serre, Géométrie algébrique et géométrie analytique, Ann. Inst. Fourier, Grenoble 6 (1955-1956), 1-42.

[41] J. Tate, Residues of differentials on curves, Ann. Scient. É.N.S. 1 (1968), 149-159.

[42] M. van der Put, The cohomology of Monsky and Washnitzer, Introductions aux cohomologies p-adiques (Luminy, 1984), Mém. Soc. Math. France 23 (1986), 33-59. 